\title{
CONTROLLING LINEAR AND SEMILINEAR SYSTEMS FORMED BY ONE ELLIPTIC AND TWO PARABOLIC PDES WITH ONE SCALAR CONTROL *
}

\author{
E. Fernández-CarA ${ }^{1}$, J. Limaco ${ }^{2}$ And S.B. De Menezes ${ }^{3}$
}

\begin{abstract}
In this paper, we prove controllability results for some linear and semilinear systems where we find two parabolic PDEs and one elliptic PDE and we act through one locally supported in space scalar control. The arguments rely on a careful analysis of the linear case and an application of an inverse function theorem. The facts that we act through a single scalar control and one of the PDEs has no time derivative are the main novelties and introduce several nontrivial difficulties.
\end{abstract}

Mathematics Subject Classification. 35B37, 35A05, 35B40.

Received June 6, 2016. Accepted June 7, 2016.

\section{INTRODUCTION AND STATEMENT OF THE PROBLEM}

Let $\Omega$ be a bounded domain of $\mathbb{R}^{N}$, with boundary $\partial \Omega$ of class $C^{2}(N \geq 1$ is an integer). We fix $T>0$ and we set $Q:=\Omega \times(0, T)$ and $\Sigma:=\Gamma \times(0, T)$. We also consider a non-empty (small) open set $\omega \subset \Omega$; as usual, $1_{\omega}$ denotes the characteristic function of $\omega$.

In this paper, we will analyze the null controllability of the parabolic-elliptic coupled systems

$$
\begin{cases}{\left[\begin{array}{c}
y_{1} \\
y_{2} \\
0
\end{array}\right]_{t}-\Delta y=A y+B v 1_{\omega}} & \text { in } Q, \\
y=0 & \text { on } \Sigma, \\
y_{1}(x, 0)=y_{1}^{0}(x), \quad y_{2}(x, 0)=y_{2}^{0}(x) & \text { in } \Omega\end{cases}
$$

and

$$
\begin{cases}{\left[\begin{array}{c}
y_{1} \\
y_{2} \\
0
\end{array}\right]_{t}-\Delta y=F(y)+B v 1_{\omega}} & \text { in } Q, \\
y=0 & \text { on } \Sigma, \\
y_{1}(x, 0)=y_{1}^{0}(x), \quad y_{2}(x, 0)=y_{2}^{0}(x) & \text { in } \Omega .\end{cases}
$$

Keywords and phrases. Null controllability, parabolic-elliptic linear and semilinear systems, Carleman estimates.

* Partially financed by MINECO, Grant MTM2013-41286-P.

1 Dpto. EDAN e IMUS, Universidad de Sevilla, Aptdo. 1160, 41080 Sevilla, Spain. cara@us.es

2 Inst. Matemática, Universidade Federal Fluminense, Valonguinho, 24020-140, Niterói, RJ, Brasil. jlimaco@vm.uff.br

3 Dpto. Matemática, Universidade Federal do Ceará, Campus do Pici - Bloco 914, 60455-760, Fortaleza, CE, Brasil. 
Here, $y=\left(y_{1}, y_{2}, y_{3}\right)^{T}$ and we have $B=e_{1}:=(1,0,0)^{T}$ or $B=e_{2}:=(0,1,0)^{T}$ or $B=e_{3}:=(0,0,1)^{T}$. In (1.1), we assume that

$$
A=\left[\begin{array}{lll}
A_{1} & A_{2} & A_{3} \\
B_{1} & B_{2} & B_{3} \\
C_{1} & C_{2} & C_{3}
\end{array}\right], \quad \text { with } A_{j}, B_{j}, C_{j} \in \mathbb{R}, \quad C_{3} \notin \sigma(-\Delta),
$$

where $\sigma(-\Delta)$ is the set of the eigenvalues of the Dirichlet Laplacian in $\Omega$. On the other hand, in (1.2), the assumptions on $F$ are the following:

$$
F=\left(F_{1}(y), F_{2}(y), F_{3}(y)\right)^{T} \text { with } F_{j} \in W^{2, \infty}\left(\mathbb{R}^{3}\right), F_{j}(0)=0(1 \leq j \leq 3) .
$$

Sometimes (but not always), we will also impose that

$$
\frac{\partial F_{3}}{\partial y_{3}}(y) \leq a<\lambda_{1} \text { for all } y \in \mathbb{R}^{3},
$$

where $\lambda_{1}$ is the first eigenvalue of the Dirichlet Laplacian in $\Omega$.

If $\left(y_{1}^{0}, y_{2}^{0}\right) \in L^{2}(\Omega) \times L^{2}(\Omega), v \in L^{2}(\omega \times(0, T))$ and $A$ (resp. the function $F$ ) satisfies (1.3) (resp. (1.4) and (1.5)), then (1.1) (resp. (1.2)) possesses exactly one weak solution $y=\left(y_{1}, y_{2}, y_{3}\right)^{T}$, with

$$
\left\{\begin{array}{l}
y_{1}, y_{2} \in L^{2}\left(0, T ; H_{0}^{1}(\Omega)\right) \cap C^{0}\left([0, T] ; L^{2}(\Omega)\right), \\
y_{1, t}, y_{2, t} \in L^{2}\left(0, T ; H^{-1}(\Omega)\right), \\
y_{3} \in L^{2}(0, T ; D(-\Delta)), \quad y_{3} \in C^{0}([0, T] ; D(-\Delta)) \text { if } B \neq e_{3}
\end{array}\right.
$$

and appropriate estimates. If we additionally have $\left(y_{1}^{0}, y_{2}^{0}\right) \in H_{0}^{1}(\Omega) \times H_{0}^{1}(\Omega)$, the following can also be affirmed:

$$
y_{1}, y_{2} \in L^{2}(0, T ; D(-\Delta)) \cap C^{0}\left([0, T] ; H_{0}^{1}(\Omega)\right), \quad y_{1, t}, y_{2, t} \in L^{2}(Q),
$$

again with appropriate estimates. The proofs of these assertions are sketched in Appendix A (see Sect. A).

Throughout this paper, $C$ denotes a generic positive constant depending on $\Omega, \omega$ and maybe other data. Sometimes, we will emphasize the fact that $C$ depends on (say) $T$ by writing $C(T)$. For all $m \geq 1$, the inner product and norm in $L^{2}(\Omega)^{m}$ will be respectively denoted by $(\cdot, \cdot)$ and $\|\cdot\|$; on the other hand, $|\cdot|$ will stand for the Euclidean norm in $\mathbb{R}^{m}$.

Definition 1.1. It will be said that (1.1) is null-controllable at time $T$ if, for any $\left(y_{1}^{0}, y_{2}^{0}\right) \in L^{2}(\Omega) \times L^{2}(\Omega)$, there exist controls $v \in L^{2}(\omega \times(0, T))$ such that the associated states satisfy

$$
y_{1}(x, T)=y_{2}(x, T)=0 \quad \text { in } \quad \Omega .
$$

A completely similar definition can be given for (1.2). Finally, it will be said that (1.2) is locally null-controllable at time $T$ if there exists $\epsilon>0$ such that, for any $\left(y_{1}^{0}, y_{2}^{0}\right) \in L^{2}(\Omega) \times L^{2}(\Omega)$ with

$$
\left\|\left(y_{1}^{0}, y_{2}^{0}\right)\right\| \leq \epsilon,
$$

there exist controls $v \in L^{2}(\omega \times(0, T))$ such that the associated states satisfy (1.8).

It will be seen later that, under the assumptions (1.3) or (1.4), we can also get information from (1.8) on the behavior of $y_{3}(\cdot, t)$ as $t \rightarrow T$; but, for the moment, we will forget this (see Rems. 2.10 and 3.4 below).

The analysis of the controllability of (1.2) is motivated by many relevant applications: reaction-diffusion systems, semiconductors modelling, chemotaxis phenomena in biology, etc. Roughly speaking, any non-scalar system of the parabolic kind for which the individual variables evolve at very different speeds may be concerned. 
However, to our best knowledge, very few results are available. See [9] and [15], where other similar parabolicelliptic systems are considered; see $[18,19]$ for Keller-Segal systems; finally, see $[7,8]$ for degenerating reactiondiffusion systems.

Recall that, in the finite-dimensional context, a linear system with constant coefficients is controllable if and only if the so called algebraic Kalman rank condition is satisfied. Accordingly, when a system is controllable at some time, it is controllable at any time.

The first goal of the present paper is to extend the Kalman rank criterion to the framework of (1.1). This will be achieved in our first main result (see Thm. 2.1).

For the proof, as usual, the null controllability of (1.1) is reformulated in terms of the observability of the adjoint system, that is given by

$$
\begin{cases}-\left[\begin{array}{c}
\varphi_{1} \\
\varphi_{2} \\
0
\end{array}\right]_{t}-\Delta \varphi=A^{*} \varphi & \text { in } Q, \\
\varphi=0 & \text { on } \Sigma, \\
\varphi_{1}(x, T)=\varphi_{1}^{T}(x), \quad \varphi_{2}(x, T)=\varphi_{2}^{T}(x) & \text { in } \Omega .\end{cases}
$$

Of course, the main difficulty found to establish this property is that only one scalar control is used in (1.1).

In our second main result, we will prove that, under some conditions, the nonlinear system (1.2) is locally null-controllable, see Theorem 2.7. Specifically, we will see that, if $N \leq 4$ and $B=e_{1}$ or $B=e_{2}$, the Kalman rank condition for a linearized version of (1.2) is a sufficient condition for the local controllability of (1.2). As commented below, for the remaining cases $\left(N \geq 5\right.$ or $\left.B=e_{3}\right)$, some maybe technical difficulties are found.

For the proof, we will employ a technique relying on the so called Liusternik's Inverse Function Theorem in Banach spaces, see [1]. The arguments are inspired by the work of Fursikov and Imanuvilov [17]. Thus, in a first step, we will consider linearized systems of the form

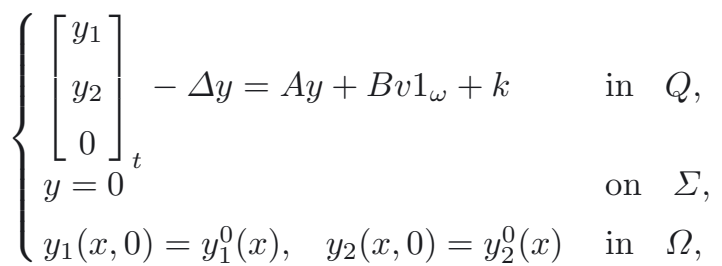

where the components of $A$ are obtained from the partial derivatives of the functions $F_{j}$ at 0 and the function $k$ decays fast enough to zero as $t \rightarrow T$. Using Theorem 2.1 and some arguments from [20], it will be seen that (1.10) is null-controllable and, moreover, one can find state-control pairs in a space $Y$ of sufficiently regular and rapidly decaying functions.

In a second step, we will rewrite the null controllability property of $(1.2)$ as an equation for $(y, v)$ in $Y$. In fact, the choice of this space is nontrivial, motivates some preliminary estimates of the null controls and associated solutions to (1.10) and deserves some work. Then, we will apply Liusternik's theorem and we will deduce the (local) desired result.

The paper is organized as follows. Section 2 deals with the linear case; more precisely, we analyze there the null controllability of systems of the kind (1.1). In Section 3, we consider the nonhomogeneous linear system (1.10) and, then, the nonlinear system (1.2). As already explained, we establish a local null controllability result. Some additional comments and open questions are indicated in Section 4. Finally, Sections A, B and C contain the proofs of several technical results. 


\section{The LINEAR CASE}

\subsection{The first main result}

We will consider the linear parabolic-elliptic coupled system (1.1), where $B=e_{1}$ or $B=e_{2}$ or $B=e_{3}$. We assume that $A$ is given by (1.3).

Recall that, for any $\left(y_{1}^{0}, y_{2}^{0}\right) \in L^{2}(\Omega) \times L^{2}(\Omega)\left(\right.$ resp. $\left.\left(y_{1}^{0}, y_{2}^{0}\right) \in H_{0}^{1}(\Omega) \times H_{0}^{1}(\Omega)\right)$ and any $v \in L^{2}(\omega \times(0, T))$, there exists exactly one weak solution to (1.1) satisfying (1.6) (resp. (1.7)).

Let us denote by $L$ the operator given by

$$
\left\{\begin{array}{l}
L=\Delta I+A: D(L) \subset L^{2}(\Omega)^{3} \mapsto L^{2}(\Omega)^{3}, \text { with } \\
D(L):=D(-\Delta)^{3}=\left[H^{2}(\Omega) \cap H_{0}^{1}(\Omega)\right]^{3} .
\end{array}\right.
$$

Then, the Kalman operator associated with $L$ and $B$ is by definition

$$
\left\{\begin{array}{l}
\mathcal{K}: D(\mathcal{K}) \subset L^{2}(\Omega)^{3} \mapsto L^{2}(\Omega)^{3}, \text { with } \\
D(\mathcal{K}):=\left\{w \in L^{2}(\Omega)^{3}:\left[L^{2} B L B \quad B\right] w \in L^{2}(\Omega)^{3}\right\} \\
\mathcal{K} w=[L \mid B] w:=\left[L^{2} B \quad L B \quad B\right] w \quad \forall w \in D(\mathcal{K})
\end{array}\right.
$$

and the (formal) adjoint of $\mathcal{K}$ is given by

$$
\left\{\begin{array}{l}
\mathcal{K}^{*}: D\left(\mathcal{K}^{*}\right) \subset L^{2}(\Omega)^{3} \mapsto L^{2}(\Omega)^{3}, \text { with } \\
D\left(\mathcal{K}^{*}\right):=\left\{\varphi \in L^{2}(\Omega)^{3}: \exists C_{\varphi}>0 \text { with }|(\mathcal{K} v, \varphi)| \leq C_{\varphi}\|v\| \quad \forall v \in D(\mathcal{K})\right\}, \\
\mathcal{K}^{*} \varphi:=\left[B^{*}\left(L^{*}\right)^{2} B^{*} L^{*} B^{*}\right]^{t} \varphi \quad \forall \varphi \in D\left(\mathcal{K}^{*}\right) .
\end{array}\right.
$$

The first main result in this paper is the following:

Theorem 2.1. The linear system (1.1) is null-controllable, with controls depending continuously on the initial data in $L^{2}(\Omega) \times L^{2}(\Omega)$, if and only if the Kalman operator $\mathcal{K}$ satisfies

$$
N\left(\mathcal{K}^{*}\right) \cap U=\{0\},
$$

where $\mathcal{K}^{*}$ is the formal adjoint of $\mathcal{K}$ and

$$
U:=\left\{\varphi=\left(\varphi_{1}, \varphi_{2}, \varphi_{3}\right)^{T}: \varphi_{3}=\left(-\Delta-C_{3} I\right)^{-1}\left(A_{3} \varphi_{1}+B_{3} \varphi_{2}\right)\right\} .
$$

The proof is given in Section 2.4 .

Let $0<\lambda_{1}<\lambda_{2} \leq \cdots$ be the eigenvalues of the Laplace-Dirichlet operator in $\Omega$, with associated eigenfunctions $\phi_{p}$. For each $p \geq 1$, let us introduce the matrices

$$
L_{p}=-\lambda_{p} I+A, \quad K_{p}=\left[L_{p}^{2} B\left|L_{p} B\right| B\right] .
$$

Also, let us denote by $P_{p}: L^{2}(\Omega)^{3} \mapsto \mathbb{R}^{3}$ the orthogonal projector associated to $\phi_{p}$ :

$$
P_{p} \psi=\left(\left(\psi_{1}, \phi_{p}\right),\left(\psi_{2}, \phi_{p}\right),\left(\psi_{3}, \phi_{p}\right)\right)^{T} \quad \forall \psi \in L^{2}(\Omega)^{3} .
$$

Then it is known that

$$
N\left(\mathcal{K}^{*}\right)=\left\{\psi \in L^{2}(\Omega)^{3}: K_{p}^{*} P_{p} \psi=0 \quad \forall p \geq 1\right\}
$$

and, consequently,

$$
N\left(\mathcal{K}^{*}\right)=\{0\} \Leftrightarrow \operatorname{det} K_{p} \neq 0 \quad \forall p \geq 1,
$$

which is equivalent to the so called Kalman condition

$$
\operatorname{rank}\left[A^{2} B|A B| B\right]=3 .
$$

For detailed proofs of these assertions, see [2]. 
Remark 2.2. In [2], it is proved that (2.2) is a necessary and sufficient condition for the null controllability of the parabolic system

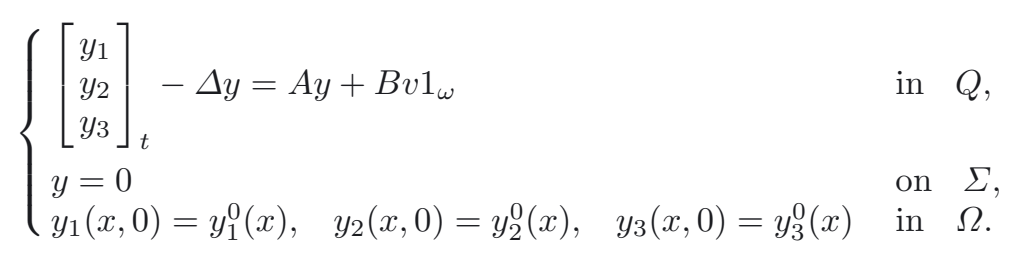

Consequently, if (2.3) is null controllable, this is also the case for (1.1). Furthermore, some simple computations show that, if $B=e_{1}$, then

$$
\operatorname{rank}\left[A^{2} B|A B| B\right]=3 \Leftrightarrow B_{1}^{2} C_{2}+B_{1} C_{1}\left(C_{3}-B_{2}\right)-B_{3} C_{1}^{2} \neq 0 .
$$

On the other hand, if $B=e_{2}$,

$$
\operatorname{rank}\left[A^{2} B|A B| \mid B\right]=3 \Leftrightarrow A_{2}^{2} C_{1}+A_{2} C_{2}\left(C_{3}-A_{1}\right)-A_{3} C_{2}^{2} \neq 0
$$

Finally, if $B=e_{3}$,

$$
\operatorname{rank}\left[A^{2} B|A B| \mid B\right]=3 \Leftrightarrow A_{3}^{2} B_{1}+A_{3} B_{3}\left(B_{2}-A_{1}\right)-A_{2} B_{3}^{2} \neq 0 .
$$

From the practical viewpoint, the following result is interesting. It is an easy consequence of Theorem 2.1 and the arguments in the proof of Lemma 2.4, see Section 2.2.

\section{Corollary 2.3.}

(a) If $B=e_{1}$, (1.1) is null controllable if and only if, for all $p \geq 1$, one has $B_{1} \lambda_{p}+B_{3} C_{1}-B_{1} C_{3} \neq 0$ or

$$
B_{3} \neq 0 \text { and } C_{1}+\left(B_{1} C_{2}+C_{1} C_{3}-B_{2} C_{1}\right) \frac{1}{\lambda_{p}-C_{3}} \neq 0
$$

(b) If $B=e_{2}$, (1.1) is null controllable if and only if, for all $p \geq 1$, one has $A_{2} \lambda_{p}+A_{3} C_{2}-A_{2} C_{3} \neq 0$ or

$$
A_{3} \neq 0 \text { and } C_{2}+\left(A_{2} C_{1}+C_{2} C_{3}-A_{1} C_{2}\right) \frac{1}{\lambda_{p}-C_{3}} \neq 0
$$

(c) Finally, if $B=e_{3},(1.1)$ is null controllable if and only if

$$
A_{3}^{2} B_{1}+A_{3} B_{3}\left(B_{2}-A_{1}\right)-A_{2} B_{3}^{2} \neq 0 .
$$

Notice in particular that, if $B=e_{3}$, the null controllability of (1.1) and (2.3) are equivalent properties. However, the situation is different for $B=e_{1}$ and $B=e_{2}$. In these cases, it may happen that (1.1) but not (2.3) be null-controllable: for example, the system (1.1) with $N=1, \Omega=(0,1), B=e_{1}$ and

$$
A=\left[\begin{array}{ccc}
A_{1} & A_{2} & A_{3} \\
1 & -2 & 1 \\
1 & 1 & -2
\end{array}\right]
$$

is null-controllable, while the corresponding parabolic system (2.3) is not. 


\subsection{Some technical results (I): Properties of $\mathcal{K}$ and $\mathcal{K}^{*}$}

Before giving the proof of Theorem 2.1, we will recall and/or establish and prove some preliminary lemmas. The first one contains several crucial properties of the Kalman operator:

Lemma 2.4. There exists $C>0$ such that:

$$
\begin{gathered}
\|\mathcal{K} v\| \leq C\left\|\Delta^{2} v\right\| \quad \forall v \in D(\mathcal{K}) \cap H^{4}(\Omega)^{3}, \\
\left\|\mathcal{K}^{*} \varphi\right\| \leq C\left\|\Delta^{2} \varphi\right\| \quad \forall \varphi \in D\left(\mathcal{K}^{*}\right) \cap H^{4}(\Omega)^{3} .
\end{gathered}
$$

Furthermore, if condition (2.1) is fulfilled, for any integer $m \geq 2$ there exists $C>0$ such that

$$
\left\|\Delta^{m-2} \varphi\right\|^{2} \leq C\left\|\Delta^{m}\left(\mathcal{K}^{*} \varphi\right)\right\|^{2} \quad \forall \varphi \in U \quad \text { with } \quad \mathcal{K}^{*} \varphi \in D\left((-\Delta)^{m}\right)^{3}
$$

Before giving the proof, let us collect some identities concerning $\mathcal{K}$ and $\mathcal{K}^{*}$.

The following is easy to prove for any $b \in \mathbb{R}^{3}$ and any $p \geq 1$ :

$$
L\left(\phi_{p} b\right)=\phi_{p}\left(L_{p} b\right), \quad \mathcal{K}\left(\phi_{p} b\right)=\phi_{p} K_{p} b
$$

From these identities, taking into account that $L$ and $\mathcal{K}$ are closed unbounded operators, a direct computation gives

$$
\begin{cases}L y=\sum_{p \geq 1} L_{p} P_{p} y \phi_{p} \quad \forall y \in D(L), \\ \mathcal{K} v=\sum_{p \geq 1} K_{p} P_{p} v \phi_{p} & \forall u \in D(\mathcal{K})\end{cases}
$$

and, consequently, we find that

$$
D(\mathcal{K})=\left\{v \in L^{2}(\Omega)^{3}: \sum_{p \geq 1}\left|K_{p} P_{p} v\right|^{2}<+\infty\right\} .
$$

In a similar way, we also get that

$$
\left\{\begin{array}{l}
\mathcal{K}^{*} \varphi=\sum_{p \geq 1} K_{p}^{*} P_{p} \varphi \phi_{p} \quad \forall \varphi \in D\left(\mathcal{K}^{*}\right) \\
D\left(\mathcal{K}^{*}\right)=\left\{\varphi \in L^{2}(\Omega)^{3}: \sum_{p \geq 1}\left|K_{p}^{*} P_{p} \varphi\right|^{2}<+\infty\right\}
\end{array}\right.
$$

Finally, we introduce the operator

$$
\left\{\begin{array}{l}
\mathcal{K} \mathcal{K}^{*}: D\left(\mathcal{K} \mathcal{K}^{*}\right) \subset L^{2}(\Omega)^{3} \mapsto L^{2}(\Omega)^{3}, \text { with } \\
D\left(\mathcal{K} \mathcal{K}^{*}\right):=\left\{\varphi \in L^{2}(\Omega)^{3}: \varphi \in D\left(\mathcal{K}^{*}\right), \mathcal{K}^{*} \varphi \in D(\mathcal{K})\right\} \\
\mathcal{K} \mathcal{K}^{*} \varphi:=\mathcal{K}\left(\mathcal{K}^{*} \varphi\right) \quad \forall \varphi \in D\left(\mathcal{K} \mathcal{K}^{*}\right)
\end{array}\right.
$$

Note that $\mathcal{K} \mathcal{K}^{*}$ is again a closed unbounded operator. A simple calculation shows that

$$
D\left(\mathcal{K} \mathcal{K}^{*}\right)=\left\{\varphi \in L^{2}(\Omega)^{3}: \sum_{p \geq 1}\left|K_{p}^{*} P_{p} \varphi\right|^{2}<+\infty, \sum_{p \geq 1}\left|K_{p} K_{p}^{*} P_{p} \varphi\right|^{2}<+\infty\right\}
$$

and

$$
\mathcal{K K}^{*} \varphi=\sum_{p \geq 1} K_{p} K_{p}^{*} P_{p} \varphi \phi_{p} \quad \forall \varphi \in D\left(\mathcal{K} \mathcal{K}^{*}\right)
$$


Proof of Lemma 2.4. The estimates (2.4) and (2.5) are proved in [2]. Let us prove (2.6).

We have:

$$
\begin{aligned}
U & =\left\{\varphi \in L^{2}(\Omega)^{3}: \varphi_{3}=\left(\Delta-C_{3}\right)^{-1}\left(A_{3} \varphi_{1}+B_{3} \varphi_{2}\right)\right\} \\
& =\left\{\varphi \in L^{2}(\Omega)^{3}: \varphi_{3}^{p}=\frac{1}{\lambda_{p}-C_{3}}\left(A_{3} \varphi_{1}^{p}+B_{3} \varphi_{2}^{p}\right) \quad \forall p \geq 1\right\},
\end{aligned}
$$

where we have denoted by $\varphi_{i}^{p}$ the $p$ th Fourier coefficient of $\varphi_{i}$.

For instance, let us assume that $B=e_{1}$. Then $\varphi \in N\left(\mathcal{K}^{*}\right) \cap U$ if and only if

$$
\left\{\begin{array}{l}
\varphi_{1}=0, \quad\left(\varphi_{3}, \phi_{p}\right)=\frac{B_{3}}{\lambda_{p}-C_{3}}\left(\varphi_{2}, \phi_{p}\right) \quad \forall p \geq 1 \\
\left(Z_{j 2}^{p}+Z_{j 3}^{p} \frac{B_{3}}{\lambda_{p}-C_{3}}\right)\left(\varphi_{2}, \phi_{p}\right)=0 \quad \forall p \geq 1, j=1,2
\end{array}\right.
$$

where the $Z_{j \ell}^{p}$ stand for the components of the matrix $K_{p}^{*}$.

Let us introduce the real numbers

$$
A_{j}^{p}:=Z_{j 2}^{p}+Z_{j 3}^{p} \frac{B_{3}}{\lambda_{p}-C_{3}}, \quad j=1,2 .
$$

Then we see that

$$
N\left(\mathcal{K}^{*}\right) \cap U=\{0\} \Leftrightarrow A_{1}^{p} \neq 0 \text { or } A_{2}^{p} \neq 0 \quad \forall p \geq 1 .
$$

After a short computation, we deduce that $\mathcal{K}_{p}^{*} \varphi=w$ if and only if

$$
A_{3}^{p} \varphi_{1}^{p}+A_{1}^{p} \varphi_{2}^{p}=F_{1}^{p}, \quad A_{4}^{p} \varphi_{1}^{p}+A_{2}^{p} \varphi_{2}^{p}=F_{2}^{p}, \quad \varphi_{1}^{p}=F_{3}^{p},
$$

where the $F_{i}^{p}$ are the components of $w$ and

$$
\left\{\begin{array}{l}
A_{3}^{p}:=\left(A_{1}-\lambda_{p}\right)^{2}+A_{2} B_{1}+A_{3} C_{1}+\frac{A_{3}}{\lambda_{p}-C_{3}}\left(C_{1}\left(A_{1}+C_{3}-2 \lambda_{p}\right)+B_{1} C_{2}\right), \\
A_{4}^{p}:=-\lambda_{p}+A_{1}+\frac{A_{3}}{\lambda_{p}-C_{3}} C_{1} .
\end{array}\right.
$$

Thus, we see that, if (2.1) holds, the components of $\varphi_{2}$ must satisfy

$$
\varphi_{2}^{p}=\frac{F_{2}^{p}}{A_{2}^{p}}-\frac{A_{4}^{p}}{A_{2}^{p}} F_{3}^{p} \text { if } A_{2}^{p} \neq 0 \text { and } \varphi_{2}^{p}=\frac{F_{1}^{p}}{A_{1}^{p}}-\frac{A_{3}^{p}}{A_{1}^{p}} F_{3}^{p} \text { if } A_{1}^{p} \neq 0 .
$$

Consequently, if $\varphi \in L^{2}(\Omega)^{3}$ and $\mathcal{K}^{*} \varphi \in D\left((-\Delta)^{m}\right)^{3}$, one has

$$
\begin{gathered}
\left|\varphi_{1}^{p}\right|^{2}+\left|\varphi_{2}^{p}\right|^{2}+\left|\varphi_{3}^{p}\right|^{2} \leq C\left(\lambda_{p}^{4}+1\right)\left(\left(F_{1}^{p}\right)^{2}+\left(F_{2}^{p}\right)^{2}+\left(F_{3}^{p}\right)^{2}\right) \\
\leq C \lambda_{p}^{4}\left(\left(F_{1}^{p}\right)^{2}+\left(F_{2}^{p}\right)^{2}+\left(F_{3}^{p}\right)^{2}\right) \quad \forall p \geq 1
\end{gathered}
$$

and

$$
\sum_{p \geq 1} \lambda_{p}^{2 m-4}\left(\left|\varphi_{1}^{p}\right|^{2}+\left|\varphi_{2}^{p}\right|^{2}+\left|\varphi_{3}^{p}\right|^{2}\right) \leq \sum_{p \geq 1} \lambda^{2 m}\left(\left(F_{1}^{p}\right)^{2}+\left(F_{2}^{p}\right)^{2}+\left(F_{3}^{p}\right)^{2}\right) .
$$

Hence, we have (2.6) in this case.

The other two cases $B=e_{2}$ and $B=e_{3}$ can be treated similarly and lead to the same conclusion.

Remark 2.5. This result can also be proved in the more general case where, in (1.1), $B$ is an arbitrary nonzero vector. The argument is essentially the same, although the computations are a little more involved. 


\subsection{Some technical results (II): Properties of the adjoint state}

As usual, the null controllability of (1.1) is equivalent to a suitable observability property for the adjoint. Accordingly, we will be concerned with the system $(1.9)$, where $\left(\varphi_{1}^{T}, \varphi_{2}^{T}\right) \in L^{2}(\Omega) \times L^{2}(\Omega)$.

The null controllability of (1.1), together with the continuity of the control in the space $L^{2}(\omega \times(0, T))$ with respect to the initial data, is equivalent to the observability inequality

$$
\|\varphi(\cdot, 0)\|^{2} \leq C(T) \iint_{\omega \times(0, T)}\left|B^{*} \varphi\right|^{2} \mathrm{~d} x \mathrm{~d} t
$$

with $C(T)$ independent of $\left(\varphi_{1}^{T}, \varphi_{2}^{T}\right) \in L^{2}(\Omega) \times L^{2}(\Omega)$.

In order to prove (2.12), we will need some (well-known) results from Fursikov and Imanuvilov [17]; see also [13]. Thus, let us introduce a new non-empty open set $\omega^{\prime}$ with $\omega^{\prime} \subset \subset \omega \subset \Omega$. The following technical result from [17] is fundamental:

Lemma 2.6. There exists a function $\alpha_{0} \in C^{2}(\bar{\Omega})$ satisfying:

$$
\left\{\begin{array}{l}
\alpha_{0}(x)>0 \quad \forall x \in \Omega, \quad \alpha_{0}(x)=0 \quad \forall x \in \partial \Omega, \\
\left|\nabla \alpha_{0}(x)\right|>0 \quad \forall x \in \bar{\Omega} \backslash \omega^{\prime} .
\end{array}\right.
$$

Let us introduce the auxiliary functions

$$
\left\{\begin{array}{l}
\beta(t):=t(T-t), \rho(x, t):=\frac{\mathrm{e}^{\lambda \alpha_{0}(x)}}{\beta(t)}, \\
\bar{\alpha}(x):=\mathrm{e}^{\nu \lambda}-\mathrm{e}^{\lambda \alpha_{0}(x)}, \eta(x, t):=\bar{\alpha}(x) / \beta(t),
\end{array}\right.
$$

where $\nu>\left\|\alpha_{0}\right\|_{L^{\infty}(\Omega)}+\log 2$ and $\lambda>0$. For any $\tau \in \mathbb{R}$ and any $s>0$, we set

$$
I(\tau, s ; \phi):=\iint_{Q}(s \rho)^{\tau-1} \mathrm{e}^{-2 s \eta}\left(\left|\phi_{t}\right|^{2}+|\Delta \phi|^{2}+(s \rho)^{2}|\nabla \phi|^{2}+(s \rho)^{4}|\phi|^{2}\right) \mathrm{d} x \mathrm{~d} t .
$$

Then the following global Carleman estimates are satisfied:

Theorem 2.7. Let $\tau \in \mathbb{R}$ be given. There exist $\widetilde{\sigma}_{0}$ and $C$, only depending on $\Omega$, $\omega^{\prime}$ and $\tau$, such that any $\phi \in L^{2}\left(0, T ; H_{0}^{1}(\Omega)\right)$ with $\phi_{t}+\Delta \phi \in L^{2}(Q)$ satisfies

$$
\begin{aligned}
I(\tau, s ; \phi) \leq & C\left(\iint_{Q}(s \rho)^{\tau} \mathrm{e}^{-2 s \eta}\left|\phi_{t}+\Delta \phi\right|^{2} \mathrm{~d} x \mathrm{~d} t\right. \\
& \left.+\iint_{\omega^{\prime} \times(0, T)}(s \rho)^{\tau+3} \mathrm{e}^{-2 s \eta}|\phi|^{2} \mathrm{~d} x \mathrm{~d} t\right)
\end{aligned}
$$

for every $s \geq \widetilde{s}_{0}:=\widetilde{\sigma}_{0}\left(T+T^{2}\right)$.

See [17] for the proof.

Let us set

$$
a^{*}:=2 \min _{\bar{\Omega}} \bar{\alpha}(x)-\max _{\bar{\Omega}} \bar{\alpha}(x), b^{*}:=4 \min _{\bar{\Omega}} \bar{\alpha}(x)-3 \max _{\bar{\Omega}} \bar{\alpha}(x), c^{*}:=\max \left(a^{*}, b^{*}\right) .
$$

The proof of Theorem 2.1 relies on the following technical result, that is established in Appendix B (see Sect. B):

Lemma 2.8. Assume that (2.1) holds. Then, for any $\tau \in \mathbb{R}$ and any integer $m \geq 2$, there exist $\tilde{\sigma}, C>0$, only depending on $\Omega, \omega, A, m$ and $\tau$, such that, for any $s \geq \tilde{\sigma}\left(T+T^{2}\right)$ and any $\left(\varphi_{1}^{T}, \varphi_{2}^{T}\right) \in L^{2}(\Omega) \times L^{2}(\Omega)$, the associated solution to (1.9) satisfies

$$
\iint_{Q}(s \rho)^{\tau} \mathrm{e}^{-2 s \eta}\left|\Delta^{m-2} \varphi\right|^{2} \mathrm{~d} x \mathrm{~d} t \leq C \iint_{\omega \times(0, T)}(s \rho)^{\tau+10 m+26} \mathrm{e}^{-\frac{2 s c^{*}}{t(T-t)}}\left|B^{*} \varphi\right|^{2} \mathrm{~d} x \mathrm{~d} t .
$$




\subsection{Proof of Theorem 2.1}

We can now achieve the proof of our first main result.

Let us begin with the necessary part. Thus, let us assume that $N\left(\mathcal{K}^{*}\right) \cap U \neq\{0\}$. Then, in view of (2.9), $A_{1}^{p}=A_{2}^{p}=0$ for some $p$.

First, let us consider the case $B=e_{1}$.

There exist final data $\left(\varphi_{1}^{T}, \varphi_{2}^{T}\right)$ such that the associated solutions to $(1.9)$ satisfy $B^{*} \varphi \equiv 0$ and $\varphi(x, 0) \not \equiv 0$. Indeed, let $\psi=\left(\psi_{1}, \psi_{2}, \psi_{3}\right)^{T}$ be the solution to the ODE system

$$
\left\{\begin{array}{l}
-\left[\begin{array}{c}
\psi_{1} \\
\psi_{2} \\
0
\end{array}\right]_{t}+\lambda_{p} \psi=A^{*} \psi \quad \text { in } \quad(0, T), \\
\psi_{1}(T)=0, \quad \psi_{2}(T)=1 .
\end{array}\right.
$$

and let us set $\varphi(x, t):=\phi_{p}(x) \psi(t)$. Then $\varphi$ fulfills the required properties:

- Obviously, $\psi(0) \neq 0$, whence $\varphi(x, 0) \not \equiv 0$.

- Also, a simple computation shows that

$$
A_{2}^{p}=-B_{1}-C_{1} \frac{B_{3}}{\lambda_{p}-C_{3}},
$$

whence the first equation in (2.15) reads

$$
-\psi_{1, t}+\lambda_{p} \psi_{1}=\left(A_{1}+\frac{C_{1} A_{3}}{\lambda_{p}-C_{3}}\right) \psi_{1}
$$

and, therefore, $\psi_{1}(t) \equiv 0$. This implies that $B^{*} \varphi \equiv 0$.

Consequently, the observability estimate (2.12) is not satisfied and (1.1) is not null-controllable.

The proof in the case $B=e_{2}$ is similar.

Finally, let us assume that $B=e_{3}$. If $A_{3} \neq 0$, we consider the solution to the ODE in (2.15), with final data $\psi_{1}(T)=-B_{3} / A_{3}, \psi_{2}(T)=1$ and we set $\varphi(x, t):=\phi_{p}(x) \psi(t)$. This way, we find again a solution to (1.1) such that $B^{*} \varphi \equiv 0$ and $\varphi(x, 0) \not \equiv 0$. A very similar construction can be performed if $B_{3} \neq 0$.

If $A_{3}=B_{3}=0$ and $N\left(\mathcal{K}^{*}\right) \cap U \neq\{0\}$, there must exist $p$ such that $\left(\varphi_{1}, \phi_{p}\right) \neq 0$ or $\left(\varphi_{2}, \phi_{p}\right) \neq 0$ and, however,

$$
K_{p}^{*}\left[\begin{array}{c}
\left(\varphi_{1}, \phi_{p}\right) \\
\left(\varphi_{2}, \phi_{p}\right) \\
0
\end{array}\right]=\left[\begin{array}{l}
0 \\
0 \\
0
\end{array}\right] .
$$

Let $\psi_{1}$ and $\psi_{2}$ solve the ODE problem

$$
\left\{\begin{array}{l}
-\left[\begin{array}{l}
\psi_{1} \\
\psi_{2}
\end{array}\right]_{t}+\lambda_{p} \psi=\left[\begin{array}{ll}
A_{1} & B_{1} \\
A_{2} & B_{2}
\end{array}\right] \psi \quad \text { in } \quad(0, T), \\
\psi_{1}(T)=1, \quad \psi_{2}(T)=0
\end{array}\right.
$$

and let us set $\psi_{3}(t) \equiv 0, \psi=\left(\psi_{1}, \psi_{2}, \psi_{3}\right)^{T}$ and $\varphi(x, t):=\phi_{p}(x) \psi(t)$. Then, again, $\varphi$ is a solution to (1.9) satisfying $B^{*} \varphi \equiv 0$ and $\varphi(x, 0) \not \equiv 0$ and, once more, (1.1) is not null-controllable.

We turn now to the sufficient part. Thus, let us assume that $(2.1)$ is satisfied and let us prove the observability inequality (2.12) for any solution to (1.9) with $\left(\varphi_{1}^{T}, \varphi_{2}^{T}\right) \in L^{2}(\Omega) \times L^{2}(\Omega)$. Let $\tilde{\sigma}$ be the constant furnished by Lemma 2.8, let us fix $s=\tilde{\sigma}\left(T+T^{2}\right)$ and let us introduce the weight

$$
\rho_{0}(t):=(s \rho)^{-23} \mathrm{e}^{\frac{s c^{*}}{t(T-t)}} .
$$


Thanks to (2.1), we can use (2.14) with $\tau=0$ and $m=2$. This gives:

$$
\iint_{Q} \mathrm{e}^{-2 s \eta^{*}}|\varphi|^{2} \mathrm{~d} x \mathrm{~d} t \leq C \iint_{\omega \times(0, T)} \rho_{0}^{-2}\left|B^{*} \varphi\right|^{2} \mathrm{~d} x \mathrm{~d} t
$$

In $\Omega \times(T / 4,3 T / 4)$, we have $2 s \eta^{*} \leq C\left(1+\frac{1}{T}\right)$. Consequently, if $B=e_{i}(1 \leq i \leq 3)$, we get:

$$
\begin{aligned}
\iint_{\Omega \times(T / 4,3 T / 4)}|\varphi|^{2} \mathrm{~d} x \mathrm{~d} t & \leq \mathrm{e}^{C(1+1 / T)} \iint_{\omega \times(0, T)} \rho_{0}^{-2}\left|B^{*} \varphi\right|^{2} \mathrm{~d} x \mathrm{~d} t \\
& =\mathrm{e}^{C(1+1 / T)} \iint_{\omega \times(0, T)} \rho_{0}^{-2}\left|\varphi_{i}\right|^{2} \mathrm{~d} x \mathrm{~d} t .
\end{aligned}
$$

From the standard energy estimates satisfied by the solution $\varphi$ to the adjoint system (1.9), we have that

$$
\left\{\begin{array}{l}
-\frac{1}{2} \frac{\mathrm{d}}{\mathrm{d} t}\left(\left\|\varphi_{1}\right\|^{2}+\left\|\varphi_{2}\right\|^{2}\right)+\left\|\nabla \varphi_{1}\right\|^{2}+\left\|\nabla \varphi_{2}\right\|^{2} \\
\leq C\left(\left\|\varphi_{1}\right\|^{2}+\left\|\varphi_{2}\right\|^{2}+\left\|\varphi_{3}\right\|^{2}\right) \\
\left\|\varphi_{3}\right\|^{2} \leq C\left(\left\|\varphi_{1}\right\|^{2}+\left\|\varphi_{2}\right\|^{2}\right)
\end{array}\right.
$$

whence we deduce that

$$
\left\|\varphi_{1}(\cdot, 0)\right\|^{2}+\left\|\varphi_{2}(\cdot, 0)\right\|^{2} \leq \mathrm{e}^{C T}\left(\left\|\varphi_{1}(\cdot, t)\right\|^{2}+\left\|\varphi_{2}(\cdot, t)\right\|^{2}\right)
$$

for all $t$. From (2.19) and (2.21), we find at once that

$$
\begin{aligned}
\left\|\varphi_{1}(\cdot, 0)\right\|^{2}+\left\|\varphi_{2}(\cdot, 0)\right\|^{2} \leq & \frac{2}{T} \mathrm{e}^{C T} \int_{T / 4}^{3 T / 4}\left(\left\|\varphi_{1}(\cdot, t)\right\|^{2}+\left\|\varphi_{2}(\cdot, t)\right\|^{2}\right) \mathrm{d} t \\
& \leq \mathrm{e}^{C(1+T+1 / T)} \iint_{\omega \times(0, T)} \rho_{0}^{-2}\left|\varphi_{i}\right|^{2} \mathrm{~d} x \mathrm{~d} t .
\end{aligned}
$$

Also, we have from the second estimate in (2.20) that

$$
\left\|\varphi_{3}(\cdot, 0)\right\|^{2} \leq C\left(\left\|\varphi_{1}(\cdot, 0)\right\|^{2}+\left\|\varphi_{2}(\cdot, 0)\right\|^{2}\right) .
$$

Accordingly, (2.12) holds and the proof is achieved.

Remark 2.9. The precise observability estimate that we have found for (1.9) is

$$
\|\varphi(\cdot, 0)\|^{2} \leq \mathrm{e}^{C(1+T+1 / T)} \iint_{\omega \times(0, T)} \rho_{0}^{-2}\left|B^{*} \varphi\right|^{2} \mathrm{~d} x \mathrm{~d} t
$$

(this will be used in Sect. 3). Thus, we see that we can find null controls for (1.1) of the form

$$
v=\rho_{0}^{-1} w, \text { with } w \in L^{2}(\omega \times(0, T))
$$

(recall that $\rho_{0}^{-1}$ decays exponentially to zero as $t \rightarrow T$ ).

Remark 2.10. If $B=e_{1}$ or $B=e_{2}$, one has $y_{3}(\cdot, t)=\left(-\Delta-C_{3} I\right)^{-1}\left(C_{1} y_{1}(\cdot, t)+C_{1} y_{1}(\cdot, t)\right)$ for all $t$. Consequently, (1.8) implies

$$
y_{3}(x, T)=0 \text { in } \Omega .
$$

The situation is different when $B=e_{3}$. However, in this case, in view of the previous Remark, we deduce that there exist controls such that one has (1.8) and

$$
y_{3}=\rho_{0}^{-1} z_{3} \text {, with } z_{3} \in L^{2}(0, T ; D(-\Delta)) .
$$

We can thus also say that $y_{3}$ vanishes at $T$ in this weak sense.

Remark 2.11. Theorem 2.1 also holds in the more general case where we assume in (1.1) tat $B$ is an arbitrary nonzero vector. The proof is essentially the same and is left to the reader. 


\section{The NONLINEAR CASE}

\subsection{The main result}

In this section, we will prove a local null controllability result for the nonlinear system (1.2), where we assume that the $F_{i}$ satisfy (1.4). Unfortunately, we cannot consider the case where the control acts on the third (elliptic) PDE; see Remark 3.5 below.

The following holds:

Theorem 3.1. Let us assume that $N \leq 4$ and $B=e_{1}$ or $B=e_{2}$. Let $\mathcal{K}$ be the Kalman operator associated to $A=F^{\prime}(0)$ and let us assume that $A$ satisfies (1.3) and $\mathcal{K}$ satisfies (2.1). Then (1.2) is locally null-controllable.

As mentioned above, we will follow for the proof a nowadays well known argument, introduced by Fursikov and Imanuvilov in [17], that relies on the following ideas:

(1) The null controllability problem for (1.2) is rewritten as a nonlinear equation of the form

$$
H(y, v)=\left(0, y_{0}\right), \quad(y, v) \in Y,
$$

where $Y$ is an appropriate Hilbert space formed by couples $(y, v)$ that (among other things) satisfy

$$
y(x, T)=0 \text { in } \Omega
$$

and $H: Y \mapsto Z$ is a $C^{1}$ mapping ( $Z$ is another Hilbert space).

(2) With these (good) definitions of $Y, Z$ and $H$, we prove that $H^{\prime}(0,0)$ is onto. This amounts to show that the linearized system (1.1) with $A=F^{\prime}(0)$ is null-controllable even if we add a non vanishing right hand side that goes sufficiently fast to zero as $t \rightarrow T$. At this point, we have to use some ideas from [20].

In these two points, we have to impose the hypotheses $N \leq 4$ and $B \neq e_{3}$; for the remaining cases, some difficulties are found.

(3) Finally, we apply Liusternik's theorem and solve (3.1) when $\left(y_{1}^{0}, y_{2}^{0}\right)$ is sufficiently small.

\subsection{The null controllability of a nonhomogeneous linear system}

Let us consider the system (1.10), where $A=F^{\prime}(0)$, the initial data $\left(y_{1}^{0}, y_{2}^{0}\right) \in L^{2}(\Omega) \times L^{2}(\Omega)$ and the right hand side $k \in L^{2}(Q)^{3}$. Recall that, by assumption, $C_{3}<\lambda_{1}$.

Our aim in this section is to find conditions on $k$ under which (1.10) is null-controllable and, also, to deduce appropriate estimates of the null controls and the associated states. The adjoint of (1.10) is given by (1.9).

If (1.1) is null-controllable at time $T>0$ and we introduce the family of null controls

$$
C_{T}:=\left\{v \in L^{2}(\omega \times(0, T)):(v, y) \text { solves }(1.1) \text { and } y(x, T) \equiv 0\right\},
$$

then the quantity

$$
E(T):=\sup _{\left\|\left(y_{1}^{0}, y_{2}^{0}\right)\right\|=1}\left(\inf _{v \in C_{T}}\|v\|_{L^{2}(\omega \times(0, T)}\right)
$$

is by definition the cost of control at time $T$.

The following holds:

$$
E: \mathbb{R}^{+} \mapsto \mathbb{R}^{+} \text {is nonincreasing and } \lim _{T \rightarrow 0^{+}} E(T)=+\infty .
$$

Indeed, if $0<T<S$, any control in $C_{T}$ belongs (after extension by zero) to $C_{S}$, whence $E(T) \geq E(S)$. On the other hand, it is clear that $E(T)$ cannot be uniformly bounded as $T \rightarrow 0^{+}$.

Let us see that

$$
E(T) \leq \mathrm{e}^{C_{0}(1+T+1 / T)} \quad \forall T>0
$$

for some $C_{0}>0$ independent of $T$. 
To this end, let us consider again the weight $\rho_{0}=\rho_{0}(t)$ given by (2.17) and, for each $\epsilon>0$, the functional $J_{\epsilon}$ defined by

$$
\begin{aligned}
J_{\epsilon}\left(\varphi_{1}^{T}, \varphi_{2}^{T}\right):= & \frac{1}{2} \iint_{\omega \times(0, T)} \rho_{0}^{-2}\left|B^{*} \varphi\right|^{2} \mathrm{~d} x \mathrm{~d} t \\
& +\epsilon\left\|\left(\varphi_{1}^{T}, \varphi_{2}^{T}\right)\right\|+\sum_{j=1}^{2}\left(\varphi_{j}(\cdot, 0), y_{j}^{0}\right),
\end{aligned}
$$

where $\varphi$ denotes the solution to (1.9) associated to $\left(\varphi_{1}^{T}, \varphi_{2}^{T}\right)$.

The function $J_{\epsilon}: L^{2}(\Omega) \times L^{2}(\Omega) \mapsto \mathbb{R}$ is continuous, strictly convex and coercive. Consequently, it possesses a unique minimizer $\left(\varphi_{1, \epsilon}^{T}, \varphi_{2, \epsilon}^{T}\right)$. Let us denote by $\varphi_{\epsilon}$ the associated solution to (1.9). We have

$$
J_{\epsilon}\left(\varphi_{1, \epsilon}^{T}, \varphi_{2, \epsilon}^{T}\right) \leq J_{\epsilon}(0,0)=0 .
$$

Therefore, we see from (2.24) that

$$
\iint_{\omega \times(0, T)} \rho_{0}^{-2}\left|B^{*} \varphi_{\epsilon}\right|^{2} \mathrm{~d} x \mathrm{~d} t \leq \mathrm{e}^{C(1+T+1 / T)}\left\|y^{0}\right\|^{2}
$$

and, introducing $v_{\epsilon}:=\left.\rho_{0}^{-2} B^{*} \varphi_{\epsilon}\right|_{\omega \times(0, T)}$, we find that

$$
\iint_{\omega \times(0, T)} \rho_{0}^{2}\left|v_{\epsilon}\right|^{2} \mathrm{~d} x \mathrm{~d} t \leq \mathrm{e}^{C(1+T+1 / T)}\left\|y^{0}\right\|^{2} .
$$

Letting $\epsilon \rightarrow 0$, we obtain a (sub)sequence of controls $v_{\epsilon}$ that converge weakly in the space $L^{2}\left(\rho_{0}^{2} ; \omega \times(0, T)\right)$ to a null control $v$ again satisfying

$$
\iint_{\omega \times(0, T)} \rho_{0}^{2}|v|^{2} \mathrm{~d} x \mathrm{~d} t \leq \mathrm{e}^{C(1+T+1 / T)}\left\|y^{0}\right\|^{2} .
$$

This proves (3.3).

A fundamental consequence of (3.3) is the following controllability result for (1.10). The proof is given in Appendix C (Sect. C):

Proposition 3.2. There exists a positive constant $R$, only depending on $\Omega, \omega$ and $A$, such that, for any $k$ satisfying

$$
\sup _{t \in[0, T]} \int_{\Omega} \mathrm{e}^{\frac{2 R}{T-t}}|k|^{2} \mathrm{~d} x<+\infty
$$

the linear system (1.10) is null-controllable. More precisely, there exists a constant $R_{0}$, again depending only on $\Omega$, $\omega$ and $A$, such that $R_{0}<R<2 R_{0}$ and, for any $\left(y_{1}^{0}, y_{2}^{0}\right) \in H_{0}^{1}(\Omega) \times H_{0}^{1}(\Omega)$, there exist state-control pairs $(y, v)$ satisfying (1.10), (1.8) and

$$
\begin{gathered}
\sup _{t \in[0, T]} \int_{\Omega} \mathrm{e}^{\frac{2 R_{0}}{T-t}}|\nabla y|^{2} \mathrm{~d} x+\iint_{Q} \mathrm{e}^{\frac{2 R_{0}}{T-t}}|\Delta y|^{2} \mathrm{~d} x \mathrm{~d} t+\iint_{\omega \times(0, T)} \mathrm{e}^{\frac{2 R_{0}}{T-t}}|v|^{2} \mathrm{~d} x \mathrm{~d} t \\
\leq C(T)\left(\sup _{t \in[0, T]} \int_{\Omega} \mathrm{e}^{\frac{2 R}{T-t}}|k|^{2} \mathrm{~d} x+\left\|\left(y_{1}^{0}, y_{2}^{0}\right)\right\|_{H_{0}^{1}(\Omega)^{2}}^{2}\right) .
\end{gathered}
$$

In the next section, this result will be used to prove the local null controllability of the nonlinear system (1.2). 


\subsection{Proof of Theorem 3.1}

First, note that it is not restrictive to assume that $\left(y_{1}^{0}, y_{2}^{0}\right) \in H_{0}^{1}(\Omega) \times H_{0}^{1}(\Omega)$. Indeed, we can initially take $v \equiv 0$ and let the system evolve to a small time $t_{0}$, with

$$
y_{j}\left(\cdot, t_{0}\right) \in H_{0}^{1}(\Omega), \quad\left\|y_{j}\left(\cdot, t_{0}\right)\right\|_{H_{0}^{1}(\Omega)} \leq C\left(t_{0}\right)\left\|\left(y_{1}^{0}, y_{2}^{0}\right)\right\|, \quad j=1,2 .
$$

Let us set $\xi \equiv \mathrm{e}^{\frac{R}{T-t}}$ and $\xi_{0} \equiv \mathrm{e}^{\frac{R_{0}}{T-t}}$, where $R$ and $R_{0}$ are the constants furnished by Proposition 3.2. Let us introduce the spaces

$$
\begin{aligned}
Y:=\{ & (y, v): v \in L^{2}(\omega \times(0, T)), \iint_{\omega \times(0, T)} \xi_{0}^{2}|v|^{2} \mathrm{~d} x \mathrm{~d} t<+\infty, \\
& y=\left(y_{1}, y_{2}, y_{3}\right)^{T}, y_{1}, y_{2} \in C^{0}\left([0, T] ; H_{0}^{1}(\Omega)\right) \cap L^{2}(0, T ; D(-\Delta)), \\
& y_{3} \in L^{\infty}\left(0, T ; H_{0}^{1}(\Omega)\right) \cap L^{2}(0, T ; D(-\Delta)), \\
& \sup _{t \in[0, T]} \xi_{0}^{2} \int_{\Omega}|\nabla y|^{2} \mathrm{~d} x+\iint_{Q} \xi_{0}^{2}|\Delta y|^{2} \mathrm{~d} x \mathrm{~d} t, \\
& +\sup _{t \in[0, T]} \xi^{2} \int_{\Omega}\left|\left[\begin{array}{c}
y_{1} \\
y_{2} \\
0
\end{array}\right]_{t}-\Delta y-F^{\prime}(0) y-B v 1_{\omega}\right|^{2} \mathrm{~d} x \\
& <+\infty\}, \\
& G:=\left\{k \in L^{\infty}\left(0, T ; L^{2}(\Omega)^{3}\right): \sup _{t \in[0, T]} \xi^{2} \int_{\Omega}|k|^{2} \mathrm{~d} x<+\infty\right\}
\end{aligned}
$$

and

$$
Z:=G \times L^{2}(\Omega)^{2} .
$$

We endow $Y$ and $Z$ with the norms $\|\cdot\|_{Y}$ and $\|\cdot\|_{Z}$, where

$$
\begin{aligned}
\|(y, v)\|_{Y}^{2}:= & \iint_{\omega \times(0, T)} \xi_{0}^{2}|v|^{2} \mathrm{~d} x \mathrm{~d} t \\
& +\sup _{t \in[0, T]} \xi_{0}^{2} \int_{\Omega}|\nabla y|^{2} \mathrm{~d} x+\iint_{Q} \xi_{0}^{2}|\Delta y|^{2} \mathrm{~d} x \mathrm{~d} t \\
& +\sup _{t \in[0, T]} \xi^{2} \int_{\Omega}\left|\left[\begin{array}{c}
y_{1} \\
y_{2} \\
0
\end{array}\right]_{t}-\Delta y-F^{\prime}(0) y-B v 1_{\omega}\right|^{2} \mathrm{~d} x
\end{aligned}
$$

and

$$
\left\|\left(k,\left(y_{1}^{0}, y_{2}^{0}\right)\right)\right\|_{Z}^{2}:=\sup _{t \in[0, T]} \xi^{2} \int_{\Omega}|k|^{2} \mathrm{~d} x+\left\|\left(y_{1}^{0}, y_{2}^{0}\right)\right\|_{H_{0}^{1}(\Omega)^{2}}^{2} .
$$

This way, $Y$ and $Z$ become Banach spaces.

Let us consider the mapping $H: Y \mapsto Z$, with

$$
H(y, v)=\left(\left[\begin{array}{c}
y_{1} \\
y_{2} \\
0
\end{array}\right]_{t}-\Delta y-F(y)-B v 1_{\omega},\left(y_{1}(\cdot, 0), y_{2}(\cdot, 0)\right)\right),
$$

where $B=e_{1}$ or $B=e_{2}$.

We will prove that there exists $\epsilon>0$ such that, if $\left(k,\left(y_{1}^{0}, y_{2}^{0}\right)\right) \in Z$ and $\left\|\left(k,\left(y_{1}^{0}, y_{2}^{0}\right)\right)\right\|_{Z} \leq \epsilon$, then the equation

$$
H(y, v)=\left(k,\left(y_{1}^{0}, y_{2}^{0}\right)\right), \quad(y, v) \in Y,
$$

possesses at least one solution. 
In particular, this will show that (1.2) is locally null-controllable and, furthermore, the state-control pairs $(y, v)$ can be found in $Y$.

We will apply the following version of Liusternik's Inverse Theorem in infinite dimensional spaces (see for instance [1]):

Theorem 3.3. Let $Y$ and $Z$ be Banach spaces and let $H: B_{r}(0) \subset Y \mapsto Z$ be a $C^{1}$ mapping. Let us assume that $H^{\prime}(0)$ is onto and let us set $\zeta_{0}=H(0)$. Then there exist $\epsilon>0$, a mapping $W: B_{\epsilon}\left(\zeta_{0}\right) \subset Z \mapsto Y$ and a constant $K>0$ satisfying:

$$
\left\{\begin{array}{l}
W(z) \in B_{r}(0) \text { and } H(W(z))=z \quad \forall z \in B_{\epsilon}\left(\zeta_{0}\right), \\
\|W(z)\|_{Y} \leq K\|z-H(0)\|_{Z} \quad \forall z \in B_{\epsilon}\left(\zeta_{0}\right) .
\end{array}\right.
$$

Let us prove that the mapping given by (3.9) satisfies the hypotheses in Theorem 3.3:

- $H: Y \mapsto Z$ is well defined and $C^{1}$.

Observe that

$$
H(y, v)=H_{0}(y, v)-(M(y, v),(0,0)) \quad \forall(y, v) \in Y,
$$

where we have introduced

$$
H_{0}(y, v):=\left(\left[\begin{array}{c}
y_{1} \\
y_{2} \\
0
\end{array}\right]_{t}-\Delta y-F^{\prime}(0) y-B v 1_{\omega},\left(y_{1}(\cdot, 0), y_{2}(\cdot, 0)\right)\right)
$$

and

$$
M(y, v):=F(y)-F^{\prime}(0) y .
$$

Accordingly, it will suffice to show that the mapping $M: Y \mapsto G$ is well defined and $C^{1}$.

First, since the $F_{j}$ belong to $W^{2, \infty}\left(\mathbb{R}^{3}\right)$, we have

$$
\left|F(y)-F^{\prime}(0) y\right| \leq\left(\sup _{s \in[0,1]}\left|F^{\prime}(s y)-F^{\prime}(0)\right|\right)|y| \leq C|y|^{2},
$$

whence

$$
\begin{aligned}
\|M(y, v)\|_{G}^{2} & =\sup _{t \in[0, T]} \xi^{2} \int_{\Omega}\left|F(y)-F^{\prime}(0) y\right|^{2} \mathrm{~d} x \mathrm{~d} t \\
& \leq C \sup _{t \in[0, T]} \xi^{2}\|y(\cdot, t)\|_{L^{4}(\Omega)}^{4} .
\end{aligned}
$$

Since $N \leq 4$, the space $H_{0}^{1}(\Omega)$ is continuously embedded in $L^{4}(\Omega)$ and, therefore, we have

$$
\begin{aligned}
\|M(y, v)\|_{G}^{2} & \leq C \sup _{t \in[0, T]} \xi^{2}\|\nabla y(\cdot, t)\|^{4} \\
& =C \sup _{t \in[0, T]} \mathrm{e}^{\frac{2 R-4 R_{0}}{T-t}} \cdot \xi_{0}^{4}\|\nabla y(\cdot, t)\|^{4} \\
& \leq C\|(y, v)\|_{Y}^{4} .
\end{aligned}
$$

Here, we have used that $R<2 R_{0}$.

This proves that $M: Y \mapsto G$ is well defined.

That $M$ is continuous can be easily deduced in a similar way; see for instance some related arguments in $[14,16]$. 
Let us now prove that $M$ is G-differentiable at any $(y, v) \in Y$ and let us compute the G-derivative $M^{\prime}(y, v)$. We suppose, for instance, that $B=e_{1}$.

For each $(y, v) \in Y$, let us introduce the linear mapping $D M(y, v)$, with

$$
(D M(y, v))\left(y^{\prime}, v^{\prime}\right)=\left(F^{\prime}(y)-F^{\prime}(0)\right) y^{\prime} \quad \forall\left(y^{\prime}, v^{\prime}\right) \in Y .
$$

Then, $D M(y, v) \in \mathcal{L}(Y ; G)$, since

$$
\begin{aligned}
\| D M(y, v) & \left(y^{\prime} v^{\prime}\right) \|_{G}^{2}=\sup _{t \in[0, T]} \xi^{2} \int_{\Omega}\left|\left(F^{\prime}(y)-F^{\prime}(0)\right) y^{\prime}\right|^{2} \mathrm{~d} x \\
& \leq C \sup _{t \in[0, T]} \xi^{2}\|y(\cdot, t)\|_{L^{4}(\Omega)}^{2}\left\|y^{\prime}(\cdot, t)\right\|_{L^{4}(\Omega)}^{2} \\
& \leq C\left(\sup _{t \in[0, T]} \xi_{0}^{2}\|\nabla y(\cdot, t)\|^{2}\right)\left(\sup _{t \in[0, T]} \xi_{0}^{2}\left\|\nabla y^{\prime}(\cdot, t)\right\|^{2}\right) \\
& \leq C\|(y, v)\|_{Y}^{2}\left\|\left(y^{\prime}, v^{\prime}\right)\right\|_{Y}^{2} .
\end{aligned}
$$

Also,

$$
\lim _{\sigma \rightarrow 0} \frac{1}{\sigma}\left[M\left((y, v)+\sigma\left(y^{\prime}, v^{\prime}\right)\right)-M(y, v)\right]=(D M(y, v))\left(y^{\prime}, v^{\prime}\right) \text { in } G
$$

for all $\left(y^{\prime}, v^{\prime}\right) \in Y$. Indeed,

$$
\begin{aligned}
& \left\|\frac{1}{\sigma}\left[M\left((y, v)+\sigma\left(y^{\prime}, v^{\prime}\right)\right)-M(y, v)\right]-(D M(y, v))\left(y^{\prime}, v^{\prime}\right)\right\|_{G}^{2} \\
& \quad=\sup _{t \in[0, T]} \xi^{2} \int_{\Omega}\left|\frac{1}{\sigma}\left[F\left(y+\sigma y^{\prime}\right)-F(y)\right]-F^{\prime}(y) y^{\prime}\right|^{2} \mathrm{~d} x \\
& \quad \leq C \sup _{t \in[0, T]} \xi^{2} \int_{\Omega}\left|\left(F^{\prime}\left(y+\theta \sigma y^{\prime}\right)-F^{\prime}(y)\right) y^{\prime}\right|^{2} \mathrm{~d} x
\end{aligned}
$$

for some measurable $\theta=\theta(x, t)$ with $0 \leq \theta \leq 1$. But this goes to zero as $\sigma \rightarrow 0$, in view of the estimates

$$
\xi^{2}\left|\left(F^{\prime}\left(y+\theta \sigma y^{\prime}\right)-F^{\prime}(y)\right) y^{\prime}\right|^{2} \leq C \xi^{2}\left|y^{\prime}\right|^{4} \sigma \leq C \xi_{0}^{4}\left|y^{\prime}\right|^{4} \sigma .
$$

We deduce that $M$ is $G$-differentiable at any $(y, v) \in Y$, with a $G$-derivative given by $D M(y, v)$.

As usual, let us denote by $M^{\prime}(y, v)$ the linear mapping defined by (3.14). Now, we shall prove that the mapping $(y, v) \mapsto M^{\prime}(y, v)$ is continuous from $Y$ into $\mathcal{L}(Y ; G)$. In other words, it will be shown that, whenever $\left(y^{n}, v^{n}\right) \rightarrow(y, v)$ in $Y$, one has

$$
\left\|\left(D M\left(y^{n}, v^{n}\right)-D M(y, v)\right)\left(y^{\prime}, v^{\prime}\right)\right\|_{G} \leq\left.\epsilon_{n}\left\|\left(y^{\prime}, v^{\prime}\right)\right\|\right|_{Y},
$$

with $\epsilon_{n} \rightarrow 0$.

The following holds:

$$
\begin{aligned}
& \left\|\left(D M\left(y^{n}, v^{n}\right)-D M(y, v)\right)\left(y^{\prime}, v^{\prime}\right)\right\|_{G}^{2}=\sup _{t \in[0, T]} \xi^{2} \int_{\Omega}\left|\left(F^{\prime}\left(y^{n}\right)-F^{\prime}(y)\right) y^{\prime}\right|^{2} \mathrm{~d} x \\
& \quad \leq C \sup _{t \in[0, T]} \xi^{2} \int_{\Omega}\left|y^{n}-y\right|^{2}\left|y^{\prime}\right|^{2} \mathrm{~d} x \\
& \quad \leq C \sup _{t \in[0, T]} \xi^{2}\left\|y^{n}(\cdot, t)-y(\cdot, t)\right\|_{L^{4}(\Omega)}^{2}\left\|y^{\prime}(\cdot, t)\right\|_{L^{4}(\Omega)}^{2} \\
& \quad \leq C\left(\sup _{t \in[0, T]} \xi_{0}^{2}\left\|\nabla y^{n}(\cdot, t)-\nabla y(\cdot, t)\right\|^{2}\right)\left(\sup _{t \in[0, T]} \xi_{0}^{2}\left\|\nabla y^{\prime}(\cdot, t)\right\|^{2}\right) \\
& \quad \leq C\left\|\left(y^{n}, v^{n}\right)-(y, v)\right\|_{Y}^{2}\left\|\left(y^{\prime}, v^{\prime}\right)\right\|_{Y}^{2} .
\end{aligned}
$$

Consequently, we certainly have (3.16) with $\epsilon_{n}=C\left\|\left(y^{n}, v^{n}\right)-(y, v)\right\|_{Y}^{2} \rightarrow 0$. 
Obviously, this implies that $M$ is continuously differentiable and, therefore, the same is also true for $H$.

- $H^{\prime}(0,0): Y \mapsto Z$ is onto.

This is obvious, thanks to the facts that that $A=F^{\prime}(0)$ satisfies (1.3) and the associated Kalman operator $\mathcal{K}$ satisfies (2.1).

Indeed, note that

$$
H^{\prime}(0,0)(y, v)=\left(\left[\begin{array}{c}
y_{1} \\
y_{2} \\
0
\end{array}\right]_{t}-\Delta y-F^{\prime}(0) y-B v 1_{\omega},\left(y_{1}(\cdot, 0), y_{2}(\cdot, 0)\right)\right) \quad \forall(y, v) \in Y
$$

Let $\left(k,\left(y_{1}^{0}, y_{2}^{0}\right)\right) \in Z$ be given. In view of Proposition 3.2, there exist couples $(y, v)$ satisfying (1.10) and (3.8). But this means that $(y, v) \in Y$ and $H^{\prime}(0,0)(y, v)=\left(k,\left(y_{1}^{0}, y_{2}^{0}\right)\right)$.

The conclusion is that the equation (3.10) can be locally solved in $Y$ and, in particular, (1.2) is locally null-controllable. This ends the proof of Theorem 2.7.

Remark 3.4. We have found a couple $(y, v)$ such that $y_{3} \in C^{0}\left([0, T] ; H_{0}^{1}(\Omega)\right)$ and

$$
y_{3}(x, T)=0 \text { in } \Omega .
$$

Indeed, recall that $B \neq e_{3}$ and one has (1.8). Therefore, in the framework of Theorem 2.7, all the components of the state vanish at $t=T$.

Remark 3.5. It does not seem easy to extend the argument in the proof of Theorem 2.7 to the cases where $N \geq 5$ or $B=e_{3}$. Let us try to explain this:

- In order to be able to apply Liusternik's theorem, we must find solutions to the linear problems

$$
H^{\prime}(0,0)(y, v)=\left(k,\left(y_{1}^{0}, y_{2}^{0}\right)\right), \quad(y, v) \in Y
$$

such that $M(y, v)$ belongs to the same space where $k$ lives; in other words, if $\xi k \in L^{r}\left(0, T ; L^{2}(\Omega)^{3}\right)$, we must be able to bound $\xi M(y, v)$ in $L^{r}\left(0, T ; L^{2}(\Omega)^{3}\right)$. In view of $(3.13)$, we would have to prove an estimate of $t \mapsto \xi\|y(\cdot, t)\|_{L^{4}(\Omega)}^{2}$ in $L^{r}(0, T)$. But it is not clear at all how to get this unless $\xi_{0}^{a} y$ belongs to $L^{2 r}\left(0, T ; L^{4}(\Omega)^{3}\right)$ for some $a>0$, which can be ensured only if $\xi_{0}^{a} y \in L^{2 r}\left(0, T ; H_{0}^{1}(\Omega)^{3}\right)$ and $H_{0}^{1}(\Omega) \hookrightarrow L^{4}(\Omega)$. From the structure of the (elliptic) PDE satisfied by $y_{3}$, it becomes clear that we need $r=+\infty$.

- If $N \geq 5$, the embedding $H_{0}^{1}(\Omega) \hookrightarrow L^{4}(\Omega)$ is not satisfied and the argument fails.

- On the other hand, if $B=e_{3}$, the right hand side of the equation satisfied by $y_{3}$ is (only) in $L^{2}(Q)$, independently of the regularity of $k_{3}$. Consequently, the desired property $\xi_{0}^{a} y \in L^{\infty}\left(0, T ; H_{0}^{1}(\Omega)^{3}\right)$ does not necessarily hold and again we cannot achieve the proof.

\section{Some AdDitional COMments AND QUESTiOns}

The controllability result in Theorem 2.1 is completely satisfactory: a necessary and sufficient criterion for the null controllability of (1.1), relatively easy to check, is given. Furthermore, Theorem 2.1 can be generalized to cover, at least, the following two situations:

- The system (1.1) with a general nonzero vector B; see Remark 2.11.

- The linear system

$$
\begin{cases}{\left[\begin{array}{c}
y_{1} \\
y_{2} \\
0
\end{array}\right]_{t}-D \Delta y=A y+B v 1_{\omega}} & \text { in } Q, \\
y=0 & \text { on } \Sigma, \\
y_{1}(x, 0)=y_{1}^{0}(x), \quad y_{2}(x, 0)=y_{2}^{0}(x) & \text { in } \Omega,\end{cases}
$$


where $D=\operatorname{diag}(\tilde{D}, 1), \tilde{D}$ is a $2 \times 2$ diagonalizable matrix satisfying

$$
\tilde{D} \xi \cdot \xi \geq \alpha|\xi|^{2} \quad \forall \xi \in \mathbb{R}^{2}, \quad \alpha>0,
$$

the matrix $A$ satisfies (1.3) and $B$ is a general nonzero vector. Indeed, after a standard change of variable, (4.1) can be equivalently rewritten as a system of the form (1.1) with a new matrix $A$ that again satisfies (1.3) and a new nonzero vector $B$.

On the other hand, we do not know at present what happens if, in (4.1), $D$ is a general $3 \times 3$ diagonalizable matrix.

Unlike Theorem 2.1, Theorem 2.7 only furnishes a partial solution to the controllability problem for (1.2): we are only able to prove a local result and, moreover, several cases are excluded. This is in contrast with the situation found in the scalar case; see $[11,12]$.

We have explained in Remark 3.5 (and also at the beginning of Sect. 3.3) that, in the argument used in the proof of Theorem 3.1, the restriction $N \leq 4$ is needed: we have to estimate the spatial $L^{4}$-norm uniformly in time and we only have estimates of this kind in the Sobolev space $H_{0}^{1}$; consequently, in order to conclude we need the previous restriction on $N$.

Unfortunately, we do not know how to avoid this. Perhaps, more regular controls give better estimates of the time derivative and Laplacian of the state in an appropriate weighted space, but this does not seem easy. Or maybe a different formulation of the null controllability problem should be investigated.

Note that, if (1.4) and (2.2) are respectively satisfied by $F$ and $A=F^{\prime}(0)$, arguing as in the proof of Theorem 2.7, it is possible to prove that the parabolic system

$$
\begin{cases}{\left[\begin{array}{l}
y_{1} \\
y_{2} \\
y_{3}
\end{array}\right]_{t}-\Delta y=F(y)+B v 1_{\omega}} & \text { in } Q, \\
y=0 & \text { on } \Sigma, \\
y_{1}(x, 0)=y_{1}^{0}(x), \quad y_{2}(x, 0)=y_{2}^{0}(x), \quad y_{3}(x, 0)=y_{3}^{0}(x) & \text { in } \Omega .\end{cases}
$$

is locally null-controllable without any restriction on $N$ and $B$.

In order to establish global results in the nonlinear case, it would be very convenient to prove before a result like Theorem 2.1 for a system of the kind (1.1) with $A$ depending on $x$ and $t$. But this does not seem a simple task; see however some related ideas in $[3,5,10]$.

Also, it is natural to consider controllability problems similar to those above with controls acting on (a part of) the boundary. This is a complex question; however, something can be said, at least when $N=1$. This will be the goal of a forthcoming paper (see $[4,6]$ for some related results).

\section{Appendix A. Well-Posedness of (1.1) And (1.2)}

For brevity, we will only sketch the proof of existence, uniqueness and regularity of the solution to (1.2). First, let us check that appropriate energy estimates hold. Indeed, we easily get from (1.2) that

$$
\begin{aligned}
\frac{1}{2} \frac{\mathrm{d}}{\mathrm{d} t}\left(\left\|y_{1}\right\|^{2}+\left\|y_{2}\right\|^{2}\right)+\sum_{i=1}^{3}\left\|\nabla y_{i}\right\|^{2} & =\sum_{i=1}^{3}\left(F_{i}(y), y_{i}\right)+\left(B v 1_{\omega}, y\right) \\
& \leq C\|y\|\left(\left\|y_{1}\right\|+\left\|y_{2}\right\|\right)+\left(F_{3}(y), y_{3}\right)+\epsilon\left\|y_{3}\right\|^{2}+C_{\epsilon}\left\|B v 1_{\omega}\right\|^{2} \\
& \leq C\|y\|\left(\left\|y_{1}\right\|+\left\|y_{2}\right\|\right)+(a+\epsilon)\left\|y_{3}\right\|^{2}+C_{\epsilon}\left\|B v 1_{\omega}\right\|^{2} \\
& \leq C_{\epsilon} \sum_{i=1}^{2}\left\|y_{i}\right\|^{2}+(a+2 \epsilon)\left\|y_{3}\right\|^{2}+C_{\epsilon}\left\|B v 1_{\omega}\right\|^{2}
\end{aligned}
$$


for all $\epsilon>0$, where $a<\lambda_{1}$. Consequently, if we take $\epsilon$ small enough, we see that

$$
\frac{\mathrm{d}}{\mathrm{d} t}\left(\left\|y_{1}\right\|^{2}+\left\|y_{2}\right\|^{2}\right)+\sum_{i=1}^{3}\left\|\nabla y_{i}\right\|^{2} \leq C \sum_{i=1}^{2}\left\|y_{i}\right\|^{2}+C\left\|B v 1_{\omega}\right\|^{2}
$$

and, from Gronwall's lemma, we deduce that $y_{1}, y_{2}$ and $y_{3}$ are bounded in $L^{2}\left(0, T ; H_{0}^{1}(\Omega)\right)$ and $y_{3}$ is bounded in $L^{\infty}\left(0, T ; L^{2}(\Omega)\right)$. From the PDEs satisfied by the $y_{i}$, we also deduce that $y_{1, t}$ and $y_{2, t}$ are bounded in $L^{2}\left(0, T ; H^{-1}(\Omega)\right)$.

As usual, these estimates suffice to get the existence of a solution satisfying (1.6).

The uniqueness of solution can be proved as follows. Let $y=\left(y_{1}, y_{2}, y_{3}\right)$ and $z=\left(z_{1}, z_{2}, z_{3}\right)$ be two solutions to (1.2) and let us set $w:=y-z$. Then

$$
\begin{aligned}
\frac{1}{2} \frac{\mathrm{d}}{\mathrm{d} t}\left(\left\|w_{1}\right\|^{2}+\left\|w_{2}\right\|^{2}\right)+\sum_{i=1}^{3}\left\|\nabla w_{i}\right\|^{2} & =\sum_{i=1}^{3}\left(F_{i}(y)-F_{i}(z), w_{i}\right) \\
& \leq C\|w\|\left(\left\|w_{1}\right\|+\left\|w_{2}\right\|\right)+\left(F_{3}(y)-F_{3}(z), w_{3}\right) \\
& \leq C\|w\|\left(\left\|w_{1}\right\|+\left\|w_{2}\right\|\right)+a\left\|w_{3}\right\|^{2} \\
& \leq C_{\epsilon} \sum_{i=1}^{2}\left\|w_{i}\right\|^{2}+(a+\epsilon)\left\|w_{3}\right\|^{2}
\end{aligned}
$$

whence

$$
\frac{\mathrm{d}}{\mathrm{d} t}\left(\left\|w_{1}\right\|^{2}+\left\|w_{2}\right\|^{2}\right)+\sum_{i=1}^{3}\left\|\nabla w_{i}\right\|^{2} \leq C_{\epsilon} \sum_{i=1}^{2}\left\|w_{i}\right\|^{2} .
$$

Again, we can use Gronwall's lemma here. This time, the conclusion is that $w_{1} \equiv w_{2} \equiv 0$ and, therefore, we also have $w_{3} \equiv 0$.

Finally, if $\left(y_{1}^{0}, y_{2}^{0}\right) \in H_{0}^{1}(\Omega) \times H_{0}^{1}(\Omega)$, the usual parabolic regularity results yield (1.7).

\section{Appendix B. Proof of Lemma 2.8}

The proof of Lemma 2.8 relies on the following result:

Lemma B.1. For any $\tau \in \mathbb{R}$ and any integer $m \geq 2$, there exist constants $\tilde{\sigma}, C>0$, only depending on $\Omega, \omega, m$ and $\tau$, with the following property: for any $s \geq \tilde{\sigma}\left(T+T^{2}\right)$ and any $\left(\varphi_{1}^{T}, \varphi_{2}^{T}\right) \in L^{2}(\Omega) \times L^{2}(\Omega)$, the corresponding solution to (1.9) satisfies

$$
\int_{0}^{T}(s \rho)^{\tau} \mathrm{e}^{-2 s \eta}\left|\Delta^{m}\left(\mathcal{K}^{*} \varphi\right)\right|_{\left[L^{2}(\Omega)\right]^{3}}^{2} \mathrm{~d} t \leq C \iint_{\omega \times(0, T)}(s \rho)^{\tau+10 m+26} \mathrm{e}^{-\frac{2 s *^{*}}{t(T-t)}}\left|B^{*} \varphi\right|^{2} \mathrm{~d} x \mathrm{~d} t .
$$

Indeed, Lemma 2.8 is an immediate consequence of Lemmas 2.4 and B.1.

Our task is thus to prove Lemma B.1. Before this, let us consider the auxiliary system

$$
\left\{\begin{array}{l}
P\left(\partial_{t}, \nabla\right) \phi=0 \text { in } \Omega \times(0, T), \\
\Delta^{m} \phi=0 \text { on } \Sigma \quad \forall m \geq 0,
\end{array}\right.
$$

where $P\left(\partial_{t}, \nabla\right)$ is the partial differential operator given by

$$
P\left(\partial_{t}, \nabla\right)=\operatorname{det}\left(\partial_{t} \tilde{I}_{2}+\lambda I+A^{*}\right), \text { with } \tilde{I}_{2}=\operatorname{diag}(1,1,0) .
$$


Notice that

$$
\left\{\begin{array}{l}
P\left(\partial_{t}, \nabla\right)=P_{3} P_{2} P_{1}+\sum_{1 \leq i_{1} \leq i_{2}<3} \alpha_{i_{1} i_{2}} P_{i_{1}} P_{i_{2}}+\sum_{i=1}^{3} \alpha_{i} P_{i}+\alpha \\
P_{1}=P_{2}=\partial_{t}+\Delta, \quad P_{3}=\Delta .
\end{array}\right.
$$

We will use the following Carleman estimate for the solutions to (B.2):

Lemma B.2. Let $\tau_{0} \in \mathbb{R}$ and the integers $k_{1}, k_{2} \geq 1$ be given. There exist $\sigma_{0}, C>0$, only depending of $\Omega, \omega$, $A, \tau_{0}, k_{1}$ and $k_{2}$, such that

$$
\sum_{\ell=0}^{k_{1}} \sum_{j=0}^{k_{2}} F\left(\tau_{0}-10(\ell+j), \Delta^{\ell} \partial_{t}^{j} \phi\right) \leq C \iint_{\omega \times(0, T)}(s \rho)^{\tau_{0}+15} \mathrm{e}^{-\frac{2 s c^{*}}{t(T-t)}}|\phi|^{2} \mathrm{~d} x \mathrm{~d} t,
$$

for any $s \geq \sigma_{0}\left(T+T^{2}\right)$ and any solution $\phi$ to (B.2) satisfying

$$
(-\Delta)^{\ell} \partial_{t}^{j} \phi \in L^{2}(0, T ; D(-\Delta)) \quad \forall \ell, j \geq 1 .
$$

In (B.4), $F(\tau, \phi)$ is given by

$$
F(\tau, \phi):=I(\tau+6, \phi)+\sum_{q=1}^{3} I\left(\tau+3, P_{q} \phi\right)+\sum_{1 \leq q \leq n \leq 3} I\left(\tau, P_{q} P_{n} \phi\right) .
$$

The proof is given in [2] for a very similar (and in some sense more complicate) system. For brevity, we will not give the details.

Now, let $\mathcal{D}$ be the linear space

$$
\mathcal{D}:=\bigcap_{p \geq 0} D\left((-\Delta)^{p}\right)
$$

(a dense subspace of $L^{2}(\Omega)$ ). We have the following.

Proposition B.3. Assume that $\left(\varphi_{1}^{T}, \varphi_{2}^{T}\right) \in \mathcal{D} \times \mathcal{D}$ and $\varphi$ is the associated solution to (1.9). Then $\varphi \in$ $C^{\ell}\left([0, T] ; D\left((-\Delta)^{p}\right)^{3}\right)$ for every $\ell, p \geq 0$. Furthermore, $\varphi_{j}$ solves $($ B.2) for $j=1,2,3$.

Proof. The change of variable $\psi(x, t)=\varphi(x, T-t)$ transforms (1.9) into

$$
\begin{cases}\psi_{1 t}-\Delta \psi_{1}=A_{1} \psi_{1}+B_{1} \psi_{2}+C_{1} \psi_{3} & \text { in } Q, \\ \psi_{2 t}-\Delta \psi_{2}=A_{2} \psi_{1}+B_{2} \psi_{2}+C_{2} \psi_{3} & \text { in } Q, \\ -\Delta \psi_{3}=A_{3} \psi_{1}+B_{3} \psi_{2}+C_{3} \psi_{3} & \text { in } Q, \\ \psi_{1}=\psi_{2}=\psi_{3}=0 & \text { on } \Sigma, \\ \psi_{1}(0)=\varphi_{01}, \psi_{2}(0)=\varphi_{02}, \psi_{3}(0)=\varphi_{03} & \text { in } \Omega .\end{cases}
$$

From (B.6) $)_{3}$, we have $\psi_{3}=\left(-\Delta-C_{3} I\right)^{-1}\left(A_{3} \psi_{1}+B_{3} \psi_{2}\right)$. Let us set $I_{2}=\operatorname{diag}(1,1)$ and let us introduce the linear mapping $\mathcal{A}: L^{2}(\Omega)^{2} \mapsto L^{2}(\Omega)^{2}$, with

$$
\left\{\begin{array}{l}
\mathcal{A}_{1}\left(\psi_{1}, \psi_{2}\right):=A_{1} \psi_{1}+B_{1} \psi_{2}+C_{1}\left(-\Delta-C_{3} I\right)^{-1}\left(A_{3} \psi_{1}+B_{3} \psi_{2}\right), \\
\mathcal{A}_{2}\left(\psi_{1}, \psi_{2}\right):=A_{2} \psi_{1}+B_{2} \psi_{2}+C_{2}\left(-\Delta-C_{3} I\right)^{-1}\left(A_{3} \psi_{1}+B_{3} \psi_{2}\right) .
\end{array}\right.
$$

Then, $\mathcal{A}$ is a bounded linear operator on $L^{2}(\Omega)^{2}$.

Since the operator $\Delta I_{2}$ is dissipative self-adjoint in $L^{2}(\Omega)^{2}$, it is the generator of an analytic semigroup. From the perturbation theory of analytic semigroups, we deduce that $\Delta I_{2}+\mathcal{A}$ is also the generator of an analytic semigroup. Since $D\left((\Delta I+\mathcal{A})^{p}\right)=D\left((-\Delta)^{p}\right)^{2}$, we find that $\psi_{1}, \psi_{2} \in C^{\ell}\left([0, T] ; D\left((-\Delta)^{p}\right)^{2}\right)$ and consequently also $\psi_{3} \in C^{\ell}\left([0, T] ; D\left((-\Delta)^{p}\right)\right)$ for all $\ell, p \geq 1$.

On the other hand, it is not difficult to check that the three components of $\varphi$ solve (B.2). 
Proof of Lemma B.1. We will first suppose that $\left(\varphi_{1}^{T}, \varphi_{2}^{T}\right) \in \mathcal{D} \times \mathcal{D}$.

Let $\varphi$ be the solution to (1.9) corresponding to these data. We can then apply Proposition B.3 and deduce that $\varphi \in C^{\ell}\left([0, T] ; D\left((-\Delta)^{p}\right)^{3}\right)$, for every $\ell, p \geq 0$, and $\varphi_{j}$ satisfies (B.2) for $1 \leq j \leq 3$. Accordingly, Lemma B.2 can be applied to $\phi=\left(B^{*} \varphi\right)_{i}$ with $k_{1}=m, k_{2}=2$ and $\tau_{0} \in \mathbb{R}$, which ensures the existence of two positive constants $\tilde{\sigma}$ and $C$ such that

$$
\sum_{\ell=0}^{m} \sum_{j=0}^{2} F\left(\tau_{0}-10(\ell+j), \Delta^{\ell} \partial_{t}^{j}\left(B^{*} \varphi\right)_{i}\right) \leq C \iint_{\omega \times(0, T)}(s \rho)^{\tau_{0}+15} \mathrm{e}^{-\frac{2 s c^{*}}{t(T-t)}}\left|\left(B^{*} \varphi\right)_{i}\right|^{2} \mathrm{~d} x \mathrm{~d} t
$$

for any $s \geq \tilde{\sigma}\left(T+T^{2}\right)$.

We observe that $\ell+j \leq m+2$ and thus $\tau:=\tau_{0}-10 m-11 \leq \tau_{0}-10(\ell+j)+9$. This implies that $(s \rho)^{\tau}=(s \rho)^{\tau_{0}-10 m-11} \leq(s \rho)^{\tau_{0}-10(\ell+j)+9}$ and also

$$
\begin{aligned}
\iint_{Q}(s \rho)^{\tau} \mathrm{e}^{-2 s \eta}\left|\Delta^{\ell} \partial_{t}^{j}\left(B^{*} \varphi\right)_{i}\right|^{2} \mathrm{~d} x \mathrm{~d} t & \leq \iint_{Q}(s \rho)^{\tau_{0}-10(\ell+j)+9} \mathrm{e}^{-2 s \eta}\left|\Delta^{\ell} \partial_{t}^{j}\left(B^{*} \varphi\right)_{i}\right|^{2} \mathrm{~d} x \mathrm{~d} t \\
& \leq I\left(\tau_{0}-10(\ell+j)+6, \Delta^{\ell} \partial_{t}^{j}\left(B^{*} \varphi\right)_{i}\right) \\
& \leq F\left(\tau_{0}-4(\ell+j), \Delta^{\ell} \partial_{t}^{j}\left(B^{*} \varphi\right)_{i}\right) .
\end{aligned}
$$

Combining (B.4) and (B.8), we get the following for all $s \geq \tilde{\sigma}\left(T+T^{2}\right)$ :

$$
\begin{aligned}
\sum_{j=0}^{2} \iint_{Q}(s \rho)^{\tau} \mathrm{e}^{-2 s \eta}\left|\Delta^{m} \partial_{t}^{j}\left(B^{*} \varphi\right)_{i}\right|^{2} \mathrm{~d} x \mathrm{~d} t & \leq C \iint_{\omega \times(0, T)}(s \rho)^{\tau_{0}+15} \mathrm{e}^{-\frac{2 s c^{*}}{t(T-t)}}\left|B^{*} \varphi\right|^{2} \mathrm{~d} x \mathrm{~d} t \\
& \leq C \iint_{\omega \times(0, T)}(s \rho)^{\tau+10 m+26} \mathrm{e}^{-\frac{2 s c^{*}}{t(T-t)}}\left|B^{*} \varphi\right|^{2} \mathrm{~d} x \mathrm{~d} t
\end{aligned}
$$

On the other hand, using (1.9) and the expression of $\mathcal{K}^{*}$, after some computations, we see that

$$
\mathcal{K}^{*} \varphi= \begin{cases}\left(\varphi_{1, t t},-\varphi_{1, t}, \varphi_{1}\right) & \text { if } B=e_{1} \\ \left(\varphi_{2, t t},-\varphi_{2, t}, \varphi_{2}\right) & \text { if } B=e_{2} \\ \left(0,0, \varphi_{3}\right) & \text { if } B=e_{3}\end{cases}
$$

Hence, replacing in (B.9), the desired inequality (B.1) is found. This concludes the proof in the case $\left(\varphi_{1}^{T}, \varphi_{2}^{T}\right) \in$ $\mathcal{D} \times \mathcal{D}$.

The general case can be easily deduced from the previous one through a standard density argument.

\section{Appendix C. proof of Proposition 3.2}

We will follow an argument inspired in the proof of Proposition 2.3 in [20]. In fact, our statement and the related estimates are more complete, but the structure of the proof is very similar.

Let $q>1$ be a real number (to be fixed below) and let us introduce the intermediate times

$$
T_{j}=\left(1-\frac{1}{q^{j}}\right) T, \text { with } j \geq 0
$$

and the functions $\gamma, \zeta$ and $\zeta_{0}$, with

$$
\gamma(t):=\mathrm{e}^{C_{1}(1+T+1 / t)}, \zeta(t):=\gamma\left((q-1) q^{-2}(T-t)\right)^{1+p}, \zeta_{0}(t):=\gamma((q-1)(T-t))^{p},
$$

where $C_{1}>C_{0}\left(C_{0}\right.$ is the constant in (3.3)) and $p>1$. 
Note that $\gamma$ is decreasing and goes to $+\infty$ as $t \rightarrow 0$. Contrarily, $\zeta$ and $\zeta_{0}$ are increasing and go to $+\infty$ as $t \rightarrow T$. Furthermore, one has

$$
\gamma\left(T_{j+2}-T_{j+1}\right) \zeta\left(T_{j}\right)^{-1}=\zeta_{0}\left(T_{j+2}\right)^{-1} \quad \forall j \geq 0 .
$$

Let $k=\left(k_{1}, k_{2}, k_{3}\right)^{T}$ be given, with $k \in L^{\infty}\left(0, T ; L^{2}(\Omega)^{3}\right)$. For each $j \geq 0$, let us consider the nonhomogeneous system

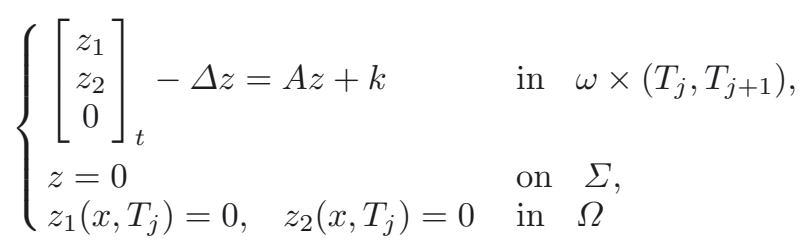

and let us denote by $z=\left(z_{1}, z_{2}, z_{3}\right)^{T}$ the associated solution. Let us set $a^{j+1}:=\left(z_{1}\left(\cdot, T_{j+1}^{-}\right), z_{2}\left(\cdot, T_{j+1}^{-}\right)\right)$for each $j \geq 0$ and $a^{0}:=\left(y_{1}^{0}, y_{2}^{0}\right)$.

On the other hand, let us introduce for each $j \geq 0$ the homogeneous controlled system

$$
\begin{cases}{\left[\begin{array}{c}
w_{1} \\
w_{2} \\
0
\end{array}\right]_{t}-\Delta w=A w+B u 1_{\omega}} & \text { in } \omega \times\left(T_{j}, T_{j+1}\right), \\
w=0 & \text { on } \Sigma, \\
\left(w_{1}, w_{2}\right)\left(x, T_{j}\right)=a^{j}(x) & \text { in } \Omega\end{cases}
$$

and let us denote by $u_{j} \in L^{2}\left(\omega \times\left(T_{j}, T_{j+1}\right)\right)$ an associated null control satisfying

$$
\left\|u_{j}\right\|_{L^{2}\left(\omega \times\left(T_{j}, T_{j+1}\right)\right)} \leq \gamma\left(T_{j+1}-T_{j}\right)\left\|a^{j}\right\| .
$$

That the controls $u_{j}$ exist is implied by the definition of $\gamma$.

It is clear that the couple $(y, v)$ defined by $y=z+w$ in each $\Omega \times\left(T_{j}, T_{j+1}\right)$ and $v=u_{j}$ in each $\omega \times\left(T_{j}, T_{j+1}\right)$ is a state-control pair satisfying (1.10). Let us prove that

$$
\begin{aligned}
& \sup _{t \in[0, T]} \zeta_{0}^{2} \int_{\Omega}|\nabla y|^{2} \mathrm{~d} x+\iint_{Q} \zeta_{0}^{2}|\Delta y|^{2} \mathrm{~d} x \mathrm{~d} t+\iint_{\omega \times(0, T)} \zeta_{0}^{2}|v|^{2} \mathrm{~d} x \mathrm{~d} t \\
& \leq C\left(\sup _{t \in[0, T]} \zeta^{2} \int_{\Omega}|k|^{2} \mathrm{~d} x+\left\|\left(y_{1}^{0}, y_{2}^{0}\right)\right\|_{H_{0}^{1}(\Omega) \times H_{0}^{1}(\Omega)}^{2}\right) .
\end{aligned}
$$

Note that the solution to (C.1) satisfies

$$
\begin{aligned}
z_{1}, z_{2} & \in C^{0}\left(\left[T_{j}, T_{j+1}\right] ; H_{0}^{1}(\Omega)^{3}\right) \cap L^{2}\left(T_{j}, T_{j+1} ; D(-\Delta)^{3}\right), \\
z_{3} & \in L^{\infty}\left(T_{j}, T_{j+1} ; H_{0}^{1}(\Omega)^{3}\right) \cap L^{2}\left(T_{j}, T_{j+1} ; D(-\Delta)^{3}\right)
\end{aligned}
$$

and

$$
\sup _{t \in[0, T]} \int_{\Omega}|\nabla z|^{2} \mathrm{~d} x+\iint_{Q}|\Delta z|^{2} \mathrm{~d} x \mathrm{~d} t \leq C \sup _{t \in\left[T_{j}, T_{j+1}\right]} \int_{\Omega}|k|^{2} \mathrm{~d} x .
$$

In particular,

$$
\left\|a^{j+1}\right\|_{H_{0}^{1}(\Omega)^{2}}^{2} \leq C \sup _{t \in\left[T_{j}, T_{j+1}\right]} \int_{\Omega}|k|^{2} \mathrm{~d} x
$$


whence we get the following estimates for $u_{j+1}$ :

$$
\begin{aligned}
\left\|u_{j+1}\right\|_{L^{2}\left(\omega \times\left(T_{j+1}, T_{j+2}\right)\right)}^{2} & \leq \gamma\left(T_{j+2}-T_{j+1}\right)^{2}\left\|a^{j+1}\right\|^{2} \\
& \leq C \gamma\left(T_{j+2}-T_{j+1}\right)^{2} \sup _{t \in\left[T_{j}, T_{j+1}\right]} \int_{\Omega}|k|^{2} \mathrm{~d} x \\
& \leq C \gamma\left(T_{j+2}-T_{j+1}\right)^{2} \zeta\left(T_{j}\right)^{-2} \sup _{t \in\left[T_{j}, T_{j+1}\right]} \int_{\Omega} \zeta^{2}|k|^{2} \mathrm{~d} x \\
& =C \zeta_{0}\left(T_{j+1}\right)^{-2} \sup _{t \in\left[T_{j}, T_{j+1}\right]} \int_{\Omega} \zeta^{2}|k|^{2} \mathrm{~d} x
\end{aligned}
$$

and then

$$
\begin{aligned}
\left\|\zeta_{0} u_{j+1}\right\|_{L^{2}\left(\omega \times\left(T_{j+1}, T_{j+2}\right)\right)} & \leq \zeta_{0}\left(T_{j+1}\right)\left\|u_{j+1}\right\|_{L^{2}\left(\omega \times\left(T_{j+1}, T_{j+2}\right)\right)} \\
& \leq C\|\zeta k\|_{L^{\infty}\left(T_{j}, T_{j+1} ; L^{2}(\Omega)^{3}\right)} .
\end{aligned}
$$

Obviously, we also have

$$
\left\|\zeta_{0} u_{0}\right\|_{L^{2}\left(\omega \times\left(0, T_{1}\right)\right)} \leq \gamma\left(T_{1}\right)\left\|\left(y_{1}^{0}, y_{2}^{0}\right)\right\| .
$$

Taking into account all this, we deduce that

$$
\left\|\zeta_{0} u_{0}\right\|_{L^{2}(\omega \times(0, T))} \leq C\left(\|\zeta k\|_{L^{\infty}\left(0, T ; L^{2}(\Omega)^{3}\right)}+\left\|\left(y_{1}^{0}, y_{2}^{0}\right)\right\|\right) .
$$

We can argue similarly with $y=z+w$ in each $\left(T_{j}, T_{j+1}\right)$. Indeed, for any $j \geq 1$ we have

$$
\begin{aligned}
\sup _{t \in\left[T_{j}, T_{j+1}\right]} & \int_{\Omega}|\nabla y|^{2} \mathrm{~d} x+\iint_{\Omega \times\left(T_{j}, T_{j+1}\right)}|\Delta y|^{2} \mathrm{~d} x \mathrm{~d} t \\
& \leq C\left(\left\|u_{j+1}\right\|_{L^{2}\left(\omega \times\left(T_{j}, T_{j+1}\right)\right)}^{2}+\|k\|_{L^{\infty}\left(T_{j}, T_{j+1} ; L^{2}(\Omega)^{3}\right)}^{2}+\left\|a^{j}\right\|_{H_{0}^{1}(\Omega)^{2}}^{2}\right) \\
& \leq C \gamma\left(T_{j+1}-T_{j}\right)^{2}\|k\|_{L^{\infty}\left(T_{j-1}, T_{j+1} ; L^{2}(\Omega)^{3}\right)}^{2} \\
& \leq C \gamma\left(T_{j+1}-T_{j}\right)^{2} \zeta\left(T_{j-1}\right)^{-2}\|\zeta k\|_{L^{\infty}\left(T_{j-1}, T_{j+1} ; L^{2}(\Omega)^{3}\right)}^{2} \\
& =C \zeta_{0}\left(T_{j+1}\right)^{-2}\|\zeta k\|_{L^{\infty}\left(T_{j-1}, T_{j+1} ; L^{2}(\Omega)^{3}\right)}^{2}
\end{aligned}
$$

and

$$
\sup _{t \in\left[T_{j}, T_{j+1}\right]} \zeta_{0}^{2} \int_{\Omega}|\nabla y|^{2} \mathrm{~d} x+\iint_{\Omega \times\left(T_{j}, T_{j+1}\right)} \zeta_{0}^{2}|\Delta y|^{2} \mathrm{~d} x \mathrm{~d} t \leq C\|\zeta k\|_{L^{\infty}\left(T_{j-1}, T_{j+1} ; L^{2}(\Omega)^{3}\right)}^{2} .
$$

For $j=0$, we have

$$
\sup _{t \in\left[0, T_{1}\right]} \int_{\Omega}|\nabla y|^{2} \mathrm{~d} x+\iint_{\Omega \times\left(0, T_{1}\right)}|\Delta y|^{2} \mathrm{~d} x \mathrm{~d} t \leq C\left(\|k\|_{L^{\infty}\left(0, T_{1} ; L^{2}(\Omega)^{3}\right)}^{2}+\left\|\left(y_{1}^{0}, y_{2}^{0}\right)\right\|_{H_{0}^{1}(\Omega)^{2}}^{2}\right) .
$$

From these estimates, we immediately obtain (C.3).

Now, taking into account the definitions of $\zeta$ and $\zeta_{0}$, we easily see that (C.3) can be rewritten in the form (3.8), with

$$
R=\frac{C_{1}(1+p) q^{2}}{q-1}, \quad R_{0}=\frac{C_{1} p}{q-1}
$$

Obviously, $R_{0}<R$; moreover, if $q$ is chosen satisfying $1<q^{2}<2 p /(1+p)$ (which is possible, since $p>1$ ), we also have $R<2 R_{0}$.

This ends the proof. 


\section{REFERENCES}

[1] V.M. Alekseev, V.M. Tikhomorov and S.V. Formin, Optimal control. Consultants Bureau, New York (1987).

[2] F. Ammar-Khodja, A. Benabdallah, C. Dupaix and M. González-Burgos, A Kalman rank condition for the localized distributed controllability of a class of linear parabolic systems. J. Evol. Equ. 9 (2009) 267-291.

[3] F. Ammar-Khodja, A. Benabdallah, M. González-Burgos and L. de Teresa, Recent results on the controllability of linear coupled parabolic problems: a survey. Math. Control Relat. Fields 1 (2011) 267-306.

[4] F. Ammar-Khodja, A. Benabdallah, M. González-Burgos and L. de Teresa, The Kalman condition for the boundary controllability of coupled parabolic systems. Bounds on biorthogonal families to complex matrix exponentials. J. Math. Pures Appl. 96 (2011) 555-590.

[5] F. Ammar-Khodja, A. Benabdallah, M. González-Burgos and L. de Teresa, Minimal time of controllability of two parabolic equations with disjoint control and coupling domains. C. R. Math. Acad. Sci. Paris 352 (2014), 391-396.

[6] A. Benabdallah, F. Boyer, M. González-Burgos, Manuel and G. Olive, Sharp estimates of the one-dimensional boundary control cost for parabolic systems and application to the $N$-dimensional boundary null controllability in cylindrical domains. SIAM J. Control Optim. 52 (2014) 2970-3001.

[7] M. Bendahmane and F.W. Chaves-Silva, Null controllability of a degenerate reaction-diffusion system in cardiac electrophisyology. C. R. Math. Acad. Sci. Paris 350 (11-12) (2012) 587-590.

[8] M. Bendahmane and F. W. Chaves-Silva, Uniform null controllability for a degenerating reaction-diffusion system approximating a simplified cardiac model, to appear.

[9] F.W. Chaves-Silva, S. Guerrero and J.-P. Puel, Controllability of fast diffusion coupled parabolic systems. Math. Control Relat. Fields 4 (2014) 465-479.

[10] J.-M. Coron and P. Lissy, Local null controllability of the three-dimensional Navier-Stokes system with a distributed control having two vanishing components. Invent. Math. 198 (2014) 833-880.

[11] A. Doubova, E. Fernández-Cara, M. González-Burgos and E. Zuazua, On the controllability of parabolic systems with a nonlinear term involving the state and the gradient. SIAM J. Control Optim. 41 (2002) 798-819.

[12] E. Fernández-Cara and E. Zuazua, Null and approximate controllability for weakly blowing up semilinear heat equations. Ann. Inst. Henri Poincaré, Anal. Non Linéaire 17 (2000) 583-616.

[13] E. Fernández-Cara and S. Guerrero, Global Carleman inequalities for parabolic systems and applications to controllability. SIAM J. Control Optim. 45 (2006) 1395-1446.

[14] E. Fernández-Cara, S. Guerrero, O.Yu. Imanuvilov and J.-P. Puel, Local exact controllability of the Navier-Stokes system. J. Math. Pures Appl. 83 (2004) 1501-1542.

[15] E. Fernández-Cara, J. Limaco and S.B. de Menezes, Null controllability for a parabolic-elliptic coupled system. Bull Braz Math Soc, New Series 44 (2013) 1-24.

[16] E. Fernández-Cara, J. Limaco and S.B. de Menezes, Theoretical and numerical local null controllability of a LadyzhenskayaSmagorinsky model of turbulence. J. Math. Fluid Mech. 17 (2015) 669-698.

[17] A. Fursikov and O.Yu. Imanuvilov, Controllability of evolution equations. Vol. 34 of Lect. Notes Ser. Seoul National University, Research Institute of Mathematics, Global Analysis Research Center, Seoul (1996).

[18] B.Z. Guo and L. Zhang, Local exact controllability of a parabolic system of chemotaxis, Preprint, School of Computational and Applied Mathematics. University of the Witwatersrand, South Africa (2012).

[19] B.-Z. Guo and L. Zhang, Local exact controllability of a parabolic system of chemotaxis. Preprint arXiv:1303.4581 (2013).

[20] Y. Liu, T. Takahashi and M. Tucsnak, Single input controllability of a simplified fluid-structure interaction model. ESAIM: COCV 19 (2013) 20-42. 